\title{
Integration of genomic copy number variations and chemotherapy-response biomarkers in pediatric sarcoma
}

Lijun Cheng ${ }^{1 \dagger}$, Pankita H. Pandya ${ }^{2,3+}$, Enze Liu ${ }^{1,4}$, Pooja Chandra ${ }^{4}$, Limei Wang ${ }^{1}$, Mary E. Murray ${ }^{3}$, Jacquelyn Carter ${ }^{3}$, Michael Ferguson ${ }^{3}$, Mohammad Reza Saadatzadeh ${ }^{2,3}$, Khadijeh Bijangi-Visheshsaraei ${ }^{2,3}$, Mark Marshall ${ }^{3}$, Lang Li $\mathrm{Li}^{1,2^{*}}$, Karen E. Pollok $2,3,5^{*}$ and Jamie L. Renbarger $2,3,4,5,6^{*}$

From The International Conference on Intelligent Biology and Medicine (ICIBM) 2018

Los Angeles, CA, USA. 10-12 June 2018

\begin{abstract}
Background: While most pediatric sarcomas respond to front-line therapy, some bone sarcomas do not show radiographic response like soft-tissue sarcomas (rhabdomyosarccomas) but do show 90\% necrosis. Though, new therapies are urgently needed to improve survival and quality of life in pediatric patients with sarcomas. Complex chromosomal aberrations such as amplifications and deletions of DNA sequences are frequently observed in pediatric sarcomas. Evaluation of copy number variations (CNVs) associated with pediatric sarcoma patients at the time of diagnosis or following therapy offers an opportunity to assess dysregulated molecular targets and signaling pathways that may drive sarcoma development, progression, or relapse. The objective of this study was to utilize publicly available data sets to identify potential predictive biomarkers of chemotherapeutic response in pediatric Osteosarcoma (OS), Rhabdomyosarcoma (RMS) and Ewing's Sarcoma Family of Tumors (ESFTs) based on CNVs following chemotherapy (OS $n=117$, RMS $n=64$, ESFTs $n=25$ tumor biopsies).

Methods: There were 206 CNV profiles derived from pediatric sarcoma biopsies collected from the public databases TARGET and NCBI-Gene Expression Omnibus (GEO). Through our comparative genomic analyses of OS, RMS, and ESFTs and 22,255 healthy individuals called from the Database of Genomic Variants (DGV), we identified CNVs (amplifications and deletions) pattern of genomic instability in these pediatric sarcomas. By integrating CNVs of Cancer Cell Line Encyclopedia (CCLE) identified in the pool of genes with drug-response data from sarcoma cell lines $(n=27)$ from Cancer Therapeutics Response Portal (CTRP) Version 2, potential predictive biomarkers of therapeutic response were identified.

(Continued on next page)
\end{abstract}

\footnotetext{
* Correspondence: Lang.Li@osumc.edu; kpollok@iu.edu; jarenbar@iu.edu ${ }^{\dagger}$ Lijun Cheng and Pankita H. Pandya contributed equally to this work. ${ }^{1}$ Department of Biomedical Informatics, College of Medicine, Ohio State University, Columbus, $\mathrm{OH}$ 43210, USA

${ }^{2}$ Herman B Wells Center for Pediatric Research, Department of Pediatrics,

School of Medicine, Indiana University, Indianapolis, IN 46202, USA

Full list of author information is available at the end of the article
}

(c) The Author(s). 2019 Open Access This article is distributed under the terms of the Creative Commons Attribution 4.0 International License (http://creativecommons.org/licenses/by/4.0/), which permits unrestricted use, distribution, and reproduction in any medium, provided you give appropriate credit to the original author(s) and the source, provide a link to the Creative Commons license, and indicate if changes were made. The Creative Commons Public Domain Dedication waiver (http://creativecommons.org/publicdomain/zero/1.0/) applies to the data made available in this article, unless otherwise stated. 
(Continued from previous page)

Results: Genes associated with survival and/recurrence of these sarcomas with statistical significance were found on long arm of chromosome 8 and smaller aberrations were also identified at chromosomes 1q, 12q and x in OS, RMS, and ESFTs. A pool of 63 genes that harbored amplifications and/or deletions were frequently associated with recurrence across OS, RMS, and ESFTs. Correlation analysis of CNVs from CCLE with drug-response data of CTRP in 27 sarcoma cell lines, 33 CNVs out of 63 genes correlated with either sensitivity or resistance to 17 chemotherapies from which actionable CNV signatures such as IGF1R, MYC, MAPK1, ATF1, and MDM2 were identified. These CNV signatures could potentially be used to delineate patient populations that will respond versus those that will not respond to a particular chemotherapy.

Conclusions: The large-scale analyses of CNV-drug screening provides a platform to evaluate genetic alterations across aggressive pediatric sarcomas. Additionally, this study provides novel insights into the potential utilization of CNVs as not only prognostic but also as predictive biomarkers of therapeutic response. Information obtained in this study may help guide and prioritize patient-specific therapeutic options in pediatric bone and soft-tissue sarcomas.

Keywords: Copy number variation, Pediatric sarcomas, Precision medicine, Prognostic biomarkers, Comparative genomic hybridization-array

\section{Background}

Sarcomas are a rare form of soft-tissue and/or bone cancers [1]. Osteosarcoma (OS), Rhabdomyosarcoma (RMS) and Ewing sarcoma family of tumors (ESFTs) are the three most common types of sarcomas that affect mostly children and teenagers, and account for approximately $15 \%$ of all childhood malignancies in the United States [2-6]. Only 30\% of relapsed/recurrent OS, RMS and ESFTs patients benefit from neoadjuvant chemotherapy [7-9]. Thus, it is imperative to identify predictive biomarkers of chemotherapeutic response in these pediatric sarcoma patients to improve prognosis and clinical outcomes. This will ultimately help to stratify patient populations that will respond to chemotherapy based on their molecular landscape.

Genetic variation is one of many characteristics of pediatric sarcomas [10-13]. It has been reported that DNA copy number variations ( $\mathrm{CNVs}$ ) and gene fusions lead to altered gene expression and eventually contribute to the development of sarcoma [10-13]. There are 55 DNA structure variation sets listed as standard clinical diagnostic biomarkers for sarcoma by the medical leader report of National Comprehensive Cancer Network (NCCN) Biomarkers Compendium [https://www.nccn.org/professionals/ biomarkers/default.aspx]. Forty-five out of 55 are fusion gene variations, while 3 of the 55 genetic alterations are CNVs. However, none of these alterations have been approved as predictive biomarkers for first line chemotherapy treatment in pediatric sarcomas [https://www.nccn.org/pro fessionals/biomarkers/default.aspx] (Additional file 1). Many of these chromosomal changes may be responsible for pediatric sarcoma progression and relapse, but the underlying cause of the large number of copy number amplifications and deletions remains unclear. Genetic alterations that serve as prognostic biomarkers for aggressive pediatric sarcomas will be investigated to also determine if they can be used as predictive biomarkers of therapeutic response.
OS is the most common primary malignant bone tumor in children and adolescents and is characterized by complex deregulated signaling $[5,7]$. Comprehensive molecular profiling of OS shows copy number amplification and overexpression of genes in chr8 and chr17p11.2-p12 that strongly correlate with OS progression and relapse $[5,7]$. Amplification of MET, CCNE1, and PDGFR $\alpha$ genes provides promising prognostic biomarkers for tailoring personalized therapies for OS patients [14]. Notably, ESFT is the second most common primary malignant bone tumor in children and adolescents. The most frequent copy number gains are observed in whole chr 8 and chr 12, long arm of chr 1. Copy number loss is commonly observed on the long arm (q) of chr 16 correlates with shorter survival in ESFTs $[15,16]$. RMS is the most common soft tissue sarcoma in children. The frequent gains and amplifications associated with short-term survival include 12q13.3-q14.1 and 8p11.1-11.2 which harbor CDK4, MYCN, GLI, MDM2, FGFR1, and FGFR4 genes $[17,18]$. Most of these biomarkers have been proposed for a specific sarcoma subtypes (OS, ESFTs, RMS) but not for all three pediatric sarcomas. These prognostic biomarkers still need to be evaluated via genome-wide studies for their role as potential predictive biomarkers of therapeutic responses across multiple pediatric sarcoma subtypes.

There is still a critical need for elucidating predictive biomarkers of therapeutic response for progressive pediatric sarcomas. To this end, prognostic biomarkers of pediatric sarcomas have the potential to also serve as predictive biomarkers of therapeutic responses, which would help guide and prioritize patient-specific therapeutic options. As mentioned above, chromosomal aberrations such as DNA copy number amplifications and deletions are frequently observed in pediatric sarcomas and can be retrospectively integrated with drug response data to ultimately allow for predictions of response to chemotherapies. For our study, we focused our efforts on exploiting high-resolution array 
Comparative Genomic Hybridization (aCGH) [19] to distinguish such pediatric sarcoma-associated CNVs pattern in OS, RMS, and ESFTs. This included comparison of chromosome bands and genes in pediatric sarcomas to, healthy population CNVs in the Database of Genomic Variants (DGV) [20]. Comprehensive literature reviews were also conducted to collect CNV amplifications and deletions of many genes from the PubMed repository which may serve as a tool for predicting clinical outcomes in all three types of sarcomas.

Genomic variations can contribute to differences in cancer cell drug responses. Systematic cell line-based platforms provide an important resource to evaluate the therapeutic efficacy of candidate anticancer agents for sarcomas harboring similar genetic alterations such as chromosomal CNVs. In the present study, we conducted a comprehensive $\mathrm{CNV}$ profile comparison between sarcoma cell lines and patient tumors. CNVs in 63 genes that serve as prognostic biomarkers of pediatric sarcomas were evaluated to determine if they correlated with sensitivity or resistance to a broad class of DNA damaging chemotherapeutic agents using Cancer Cell Line Encyclopedia (CCLE) [21] and The Cancer Therapeutics Response Portal (CTRP) Version 2 [22]. The correlation of genetic alterations such as $\mathrm{CNVs}$ and response to standard-of-care agents offers the opportunity to identify potential prognostic and/or predictive biomarkers of therapeutic response that may facilitate the stratification of patients with responder versus non-responder signatures.

\section{Samples and clinical data}

Two hundred six DNA copy number profiles for pediatrics sarcoma were collected from the publicly accessible databases, NCBI - Gene Expression Omnibus (GEO) [https:// www.ncbi.nlm.nih.gov/sites/GDSbrowser/] and Therapeutically Applicable Research to Generate Effective Treatments (TARGET) [https://ocg.cancer.gov/programs/target]. These data sets included CNVs from OS $(n=117)$, RMS $(n=64)$, and ESFTs $(n=25)$ (Table 1, Additional file 2). Database of Genomic Variants (DGV) [20] specifically, the hg38 DGV provided comprehensive genomic structure variation of healthy individuals for the sarcoma CNV comparison. $\mathrm{CNVs}$ of all sarcoma tumors were tested prior to surgery without prior chemotherapy. The median age at diagnosis was 15 years (range 2-20 years). These sarcomas were intermediate to high grade $(93 \%)$. The detailed clinical sample annotation is listed in Additional file 2.

CCLE project provides a detailed genetic characterization of a large panel of human cancer cell lines $(n=947)$ which includes 24 cancer types [21]. Copy number profiles of 27-sarcoma cancer cells lines previously obtained by Affymetrix SNP Array 6.0 were collected (see Table 2 for cell lines). The sensitivity of drug responses were quantified using the Area Under the Curve (AUC) for 481 candidate cancer drugs in 27 sarcoma cell lines collected from the Cancer Therapeutics Response Portal (CTRP v2.0) [22] and integrated with copy number alterations from the CCLE.

\section{Results}

\section{Comparative analysis of CNVs from OS, RMS, and ESFTs} to healthy population genomes

A comprehensive assessment of CNVs using high-resolution array CGH (Affymetrix SNP) array was completed on OS, RMS and ESFT sarcoma patients (Fig. 1). Genes or regions frequently comprised of these CNVs were identified by comparing whole genome CNVs of a healthy population from DGV 22,255 samples.

$\mathrm{CNV}$ analyses and stratification based on amplification and/or deletion frequencies were conducted for OS, RMS, and ESFTs (Fig. 2a-c). Hierarchy clustering analyses for delineating the pattern of genomic CNVs were conducted to stratify sarcoma patients based on their relapse and metastasis status where CNV distributions greater than the $85 \%$ range represented amplification $(\mathrm{OS}=2.710$, ESFTs $=0.147$, RMS $=0.7)$ and less than the $15 \%$ range signified deletion $(\mathrm{OS}=1.414$, ESFTs $=-$ $0.1467, \mathrm{RMS}=-1.213$ ) (Fig. 2a-c). CNVs that were in between these thresholds for each sarcoma type were considered as having no change in CNVs.

OS $(n=117)$ had the most common gain (copy number amplification) in chromosomes $8,12,21$, and $\mathrm{X}$, while the most common loss (copy number deletion) was found in chromosomes 2, 10, and 13 (Fig. 2 A1). Combination of copy number amplification and deletion were observed in chromosomes 1,10 and 12 which are comprised of genes amplified or deleted in OS pathogenesis

Table 1 Datasets and their source for healthy and pediatric sarcoma patients

\begin{tabular}{llll}
\hline Data Type & Source & Platform & Sample size \\
\hline Healthy Individuals & DGV (Database of Genomic Variants) [13] & BAC Acgh, FISH, OligoACGH, PCR, Sequencing, & 22, 255 \\
& & SNP array and Digital array & 32 \\
Osteosarcoma (OS) & GEO (GSE33383) & Affymetrix Genome-Wide Human SNP & 85 \\
& TARGET & 6.0 Array chips, GPL6801 & 7 \\
Rhabdomyosarcoma (RMS) & GEO (GSE24715) & Affymetrix Mapping 250 K Sty2 SNP chips, GPL3720 & \\
& & Affymetrix Human Mapping 50 K Xba240 SNP chips, GPL2005 & 57 \\
Ewing Sarcoma (ESFTs) & GEO (GSE8398) & Agilent-013282 Human Genome CGH Microarray 44B, GPL2879 25 & 25 \\
\hline
\end{tabular}


Table 2 Sarcoma cancer cell lines

\begin{tabular}{lllll}
\hline Tissue & Histology & Cell_line & Tissue & Histology \\
\hline soft_tissue & Rhabdomyosarcoma & A-204 & bone & Ewings_sarcoma \\
soft_tissue & Rhabdomyosarcoma & Hs 729 & bone & Ewings_sarcoma \\
soft_tissue & Rhabdomyosarcoma & KYM-1 & bone & Ewings_sarcoma \\
soft_tissue & Rhabdomyosarcoma & RD & bone & Ewings_sarcoma \\
soft_tissue & Rhabdomyosarcoma & RH-30 & bone & Osteosarcoma \\
soft_tissue & Rhabdomyosarcoma & RH-41 & bone & Osteosarcoma \\
soft_tissue & Rhabdomyosarcoma & SJRH30 & Osteosarcoma \\
soft_tissue & Rhabdomyosarcoma & TE 441.T & bone & Osteosarcoma \\
soft_tissue & Rhabdomyosarcoma & TE 617.T & bone & Osteosarcoma \\
bone & Ewings_sarcoma & A-673 & bone & Osteosarcoma \\
bone & Ewings_sarcoma & CADO-ES1 & bone & Osteosarcoma \\
bone & Ewings_sarcoma & Hs 822.T & bone & Osteosarcoma \\
bone & Ewings_sarcoma & Hs 863.T & bone & Osteosarcoma \\
bone & Ewings_sarcoma & MHH-ES-1 & & Hs 888.T \\
\hline
\end{tabular}

such as RAD21, MYC, PTEN, IGF1R, and TP53 (Fig. 2 A2) $[5,7,10,11,23-26]$ (details in Additional file 3: Table S1). Frequency analyses of CNV amplifications and deletions in the healthy population indicated the existence of CNVs in regions such as 1q21, 10p11, and 15q25 (Fig. 2 A1).
ESFTs $(n=25)$ also exhibited the presence of copy number gains in chromosomes 1, 8, and 12 (Fig. 2 B1). Deletions (copy number loss) were found in chromosomes 10, 11, and X (Fig. 2 B1). Smaller aberrations were found at chromosome regions: 11q24, 22q12, 5p,

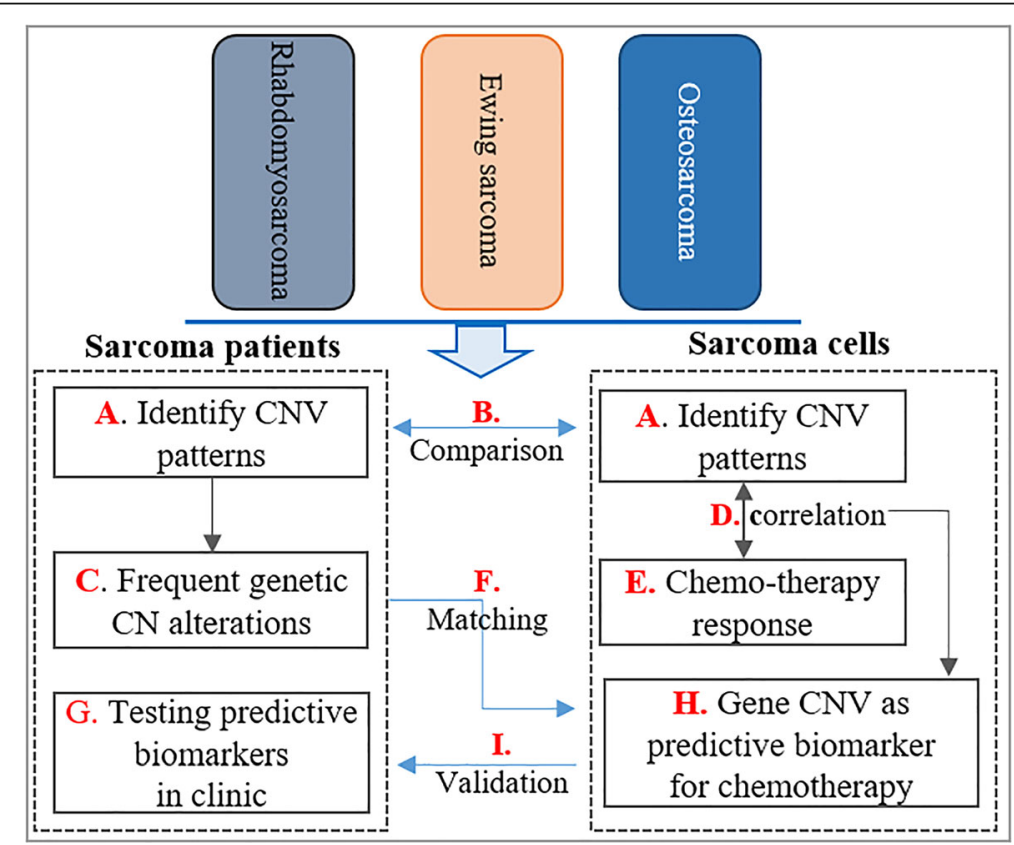

Fig. 1 Integration of genomic CNVs to chemotherapy-response for identification of predictive biomarkers of therapeutic response in pediatric sarcoma tumor biopsies and cell lines. a CNV patterns were detected in 206 sarcoma (OS, RMS, and ESFTs) patients as well as in 27 sarcoma cell lines (OS, RMS, and ESFTs). b The CNVs identified from sarcoma cells were also compared with the profile observed in the 206 sarcoma patients. c Frequent CNVs were identified by literature review from PUBMED and compared with 206 patients CNVs. d,e Large screening to evaluate drug response associated with CNVs using a Pearson Correlation calculation was completed to identify potential predictive biomarkers of therapeutic response in these sarcomas. $\mathbf{f}$ Matching sarcoma patients CNVs to sarcoma cells. g, h, i Significant predictive biomarkers of sensitivity and resistance to chemotherapy are obtained. Significant predictive biomarkers of sensitivity and resistance to chemotherapy will be identified for further exploration 

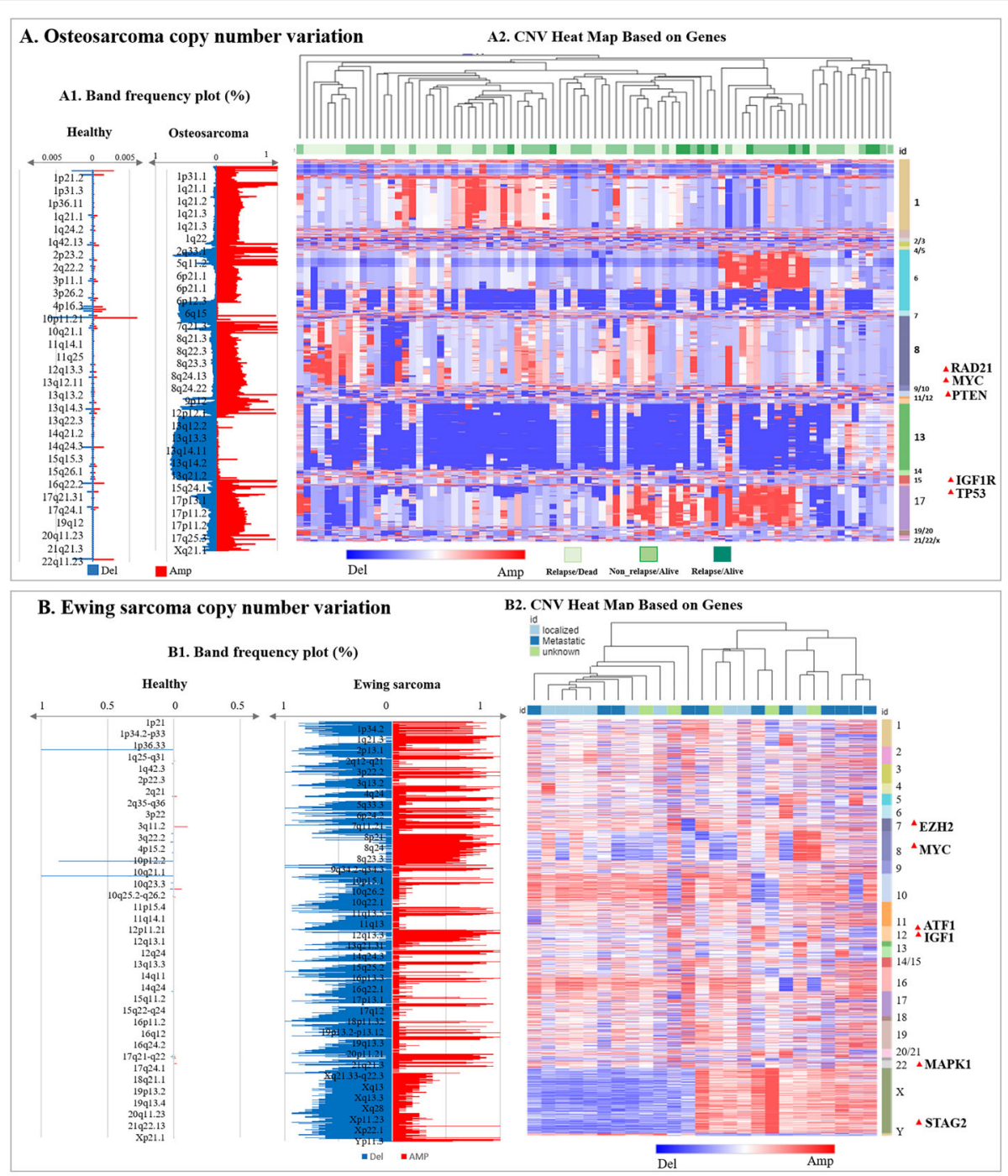

\begin{tabular}{ll} 
C. Rhabdomyosarcoma copy number variation $\quad$ C2. CNV Heat Map Based on Genes \\
\hline
\end{tabular}
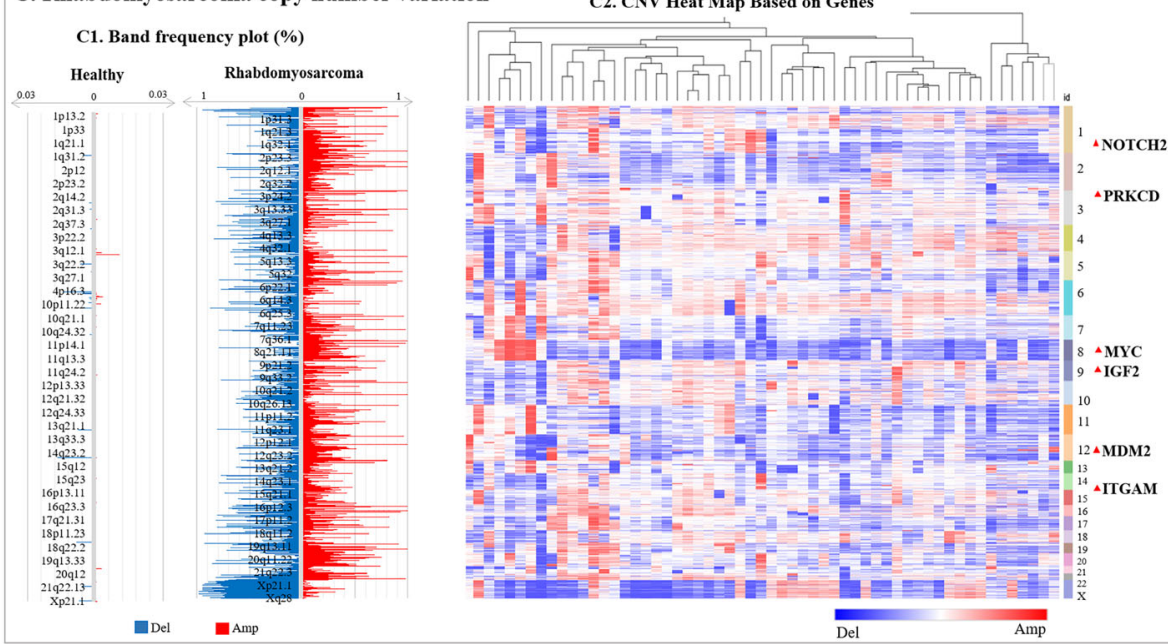

Fig. 2 (See legend on next page.) 


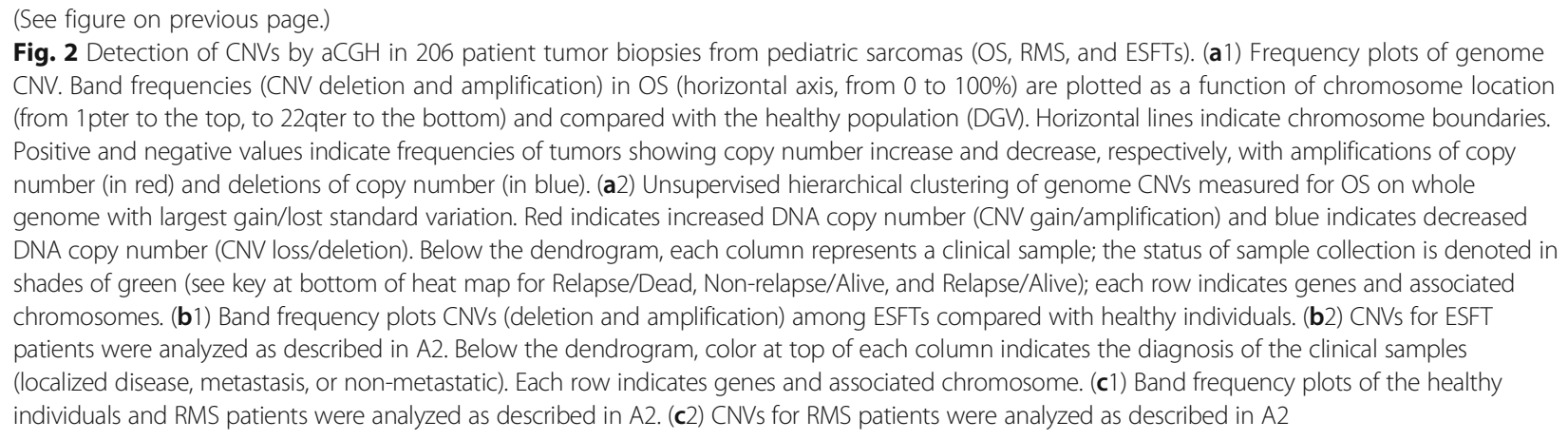

7q, and 9p (Fig. 2 B1). Some genes associated with recurrent ESFT included EZH2, MYC, ATF1, IGF1, MAPK1, FGFR1 and STAG2 (Fig. 2 B2) [2, 4, 6, 9, 27-29].

In RMS $(n=64)$, amplifications (copy number gains) were found in chromosomes 2, 8, 12, and 20. (Fig. 2 C1). Whereas, recurrent loss of heterozygosity ( $\mathrm{LOH})$ of chromosomes 1, 7, 14, and X was detected (Fig.2 C1). Genes of interest in RMS that were amplified or deleted and may contribute to the disease pathogenesis/progression included NOTCH2, PRKCD, MYC, IGF2, MDM2 and ITGAM (Fig. 2 C2) [3, 17, 18, 30, 31]. The details are shown in Additional file 3: Table S3.

There are 5417 overlapping genes among OS, ESFTs and RMS at the whole genome level compared to normal healthy controls (Fig. 3). The loss of heterozygosity (LOH) of chromosomes $1,7,14$, and $\mathrm{X}$ was detected in OS, ESFTs and RMS respectively (Additional file 4). A common pattern of copy number gains in chromosome 8 and 12 was found in OS, RMS and ESFT. The specific segment that was amplified in chromosome band 8q23-q24 included MYC, PMP1, ODF1, TRPS1, RAD21, SQLE, FAM49B and LRRC6. Moreover, MYC, which is located in $8 \mathrm{q} 24.21$, showed the highest amplification frequency of 0.78 in OS, 0.69 in ESFTs, and was not amplified in RMS with a frequency of 0.32 . However, the pattern of $\mathrm{CNV}$ exhibited differences among OS, RMS and ES on chromosome 1. The gene SELL had increased copy number amplifications in OS compared to ESFT and RMS. Transcription factor genes on chromosome 1 such as NOTCH2 (deletion), PRKAB2 (amplification) and SELL (amplification) also shared similar copy number alterations in all three types of sarcomas (Figs. 3 and 4).

\section{Significant CNVs associated with prognostic biomarkers of pediatric sarcomas (OS, RMS, ESFTs)}

Based on extensive literature review of all three sarcomas [2-22], [https://www.ncbi.nlm.nih.gov/sites/GDSbrowser/], [23-37], functional outcome of the most frequent CNVs associated with poor outcome was compiled. These gene sets for each sarcoma type is listed in Additional file 3. The top 63 frequently amplified or deleted genes in OS, RMS and ESFTs were previously shown to be associated with recurrent in OS, RMS and ESFTs and are shown in Table 3. All of genes were annotated and mapped to chromosome level by Hg19. See methods section for details on data analysis [38, 39], [http://www.affymetrix.com/ support/technical/byproduct.affx?product=500k], [40, 41]. In addition, druggable targets denoted in DrugBank (https://www.drugbank.ca/) are included.

\section{Comparison of CNVs between patient sarcoma tumors and sarcoma cell lines}

As described above, 63 genes that serve as potential prognostic biomarkers were extracted from bone and soft tissue sarcoma cell lines described in the CCLE where CNVs were categorized based on their high frequencies of amplifications and deletions (Table 2). A hierarchy clustering was used to identify cluster patterns across bone and soft tissue sarcoma cell lines (Fig. 5, left panel). Associated CNV amplification frequencies in sarcoma patient samples were compared with sarcoma cell lines (Fig. 5, right panel). EGFR amplification was showed to be amplified in $25 \%$ of the patient samples and in $24 \%$ of the sarcoma cell lines. However, MYC was amplified in $60 \%$ of the OS and ESFT patient samples while but it was only amplified in $8 \%$ of the sarcoma cell lines. IGF1R was amplified more frequencely in OS patients $31.3 \%$ than in RMS $13 \%$ and ESFT $12.5 \%$.

\section{Linking CNV profiles in pediatric sarcoma samples to drug sensitivity in sarcoma cell lines with similar CNV profiles}

To elucidate if the CNVs identified in pediatric sarcomas, it could be used to guide selection of therapies that will improve clinical outcome. We next investigated the extent of drug sensitvity in the sarcoma cell lines based on CNVs (CCLE, Table 2). Drug sensitivity correlated with amplifications and/or deletions frequently found in the pool of 63 genes harboring CNVs (Table 2). The database CTRP provides drug-response data of sarcoma cells. Therefore, evaluation and comparison of drug-response with the identified CNVs was integrated from the cell line CCLE 


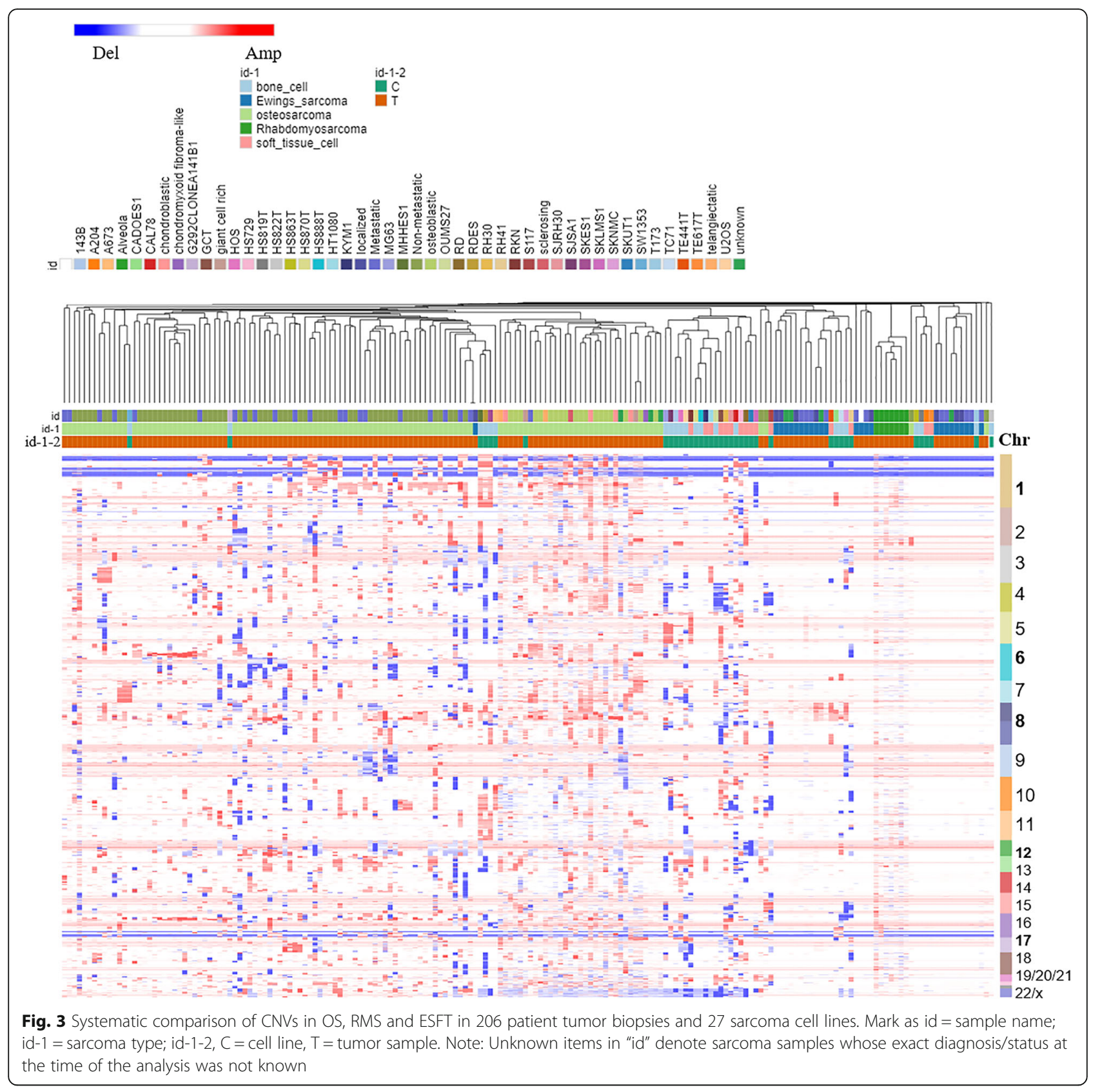

and the drug response CTRP databases. Pearson Correlation analysis between CCLE and CTRP indicated that 33 CNVs from 27 sarcoma cell lines had a positive and/or negative correlation with drug response to 17 DNA damaging agents (Fig. 6). For example, IGF1R copy number amplification correlated with sensitivity to clofarabine (Fig. 6a, see left panel), and therefore, may serve as a "sensitive" biomarker of therapeutic response to clofarabine. Since lower concentrations of drug were needed to inhibit growth of the sarcoma cell lines with IGFR1 copy number amplications [see right panel that compares AUC of clofarabine in cell lines with IGF1R gene deletion (clofarabine nonsensitive) or amplification (clofarabine sensitive)]. The significant correlation between clofarabine response associated with CNVs in 27 sarcoma cell lines is illustrated in Fig. 6b. Overall, a number of therapeutic predictive biomarkers were found (Fig. 6c). Integration of $33 \mathrm{CNVs}$ with drug response data in sarcoma cell lines uncovered differential sensitivities to commonly used chemotherapeutic drugs.

\section{Discussion}

Pediatric sarcomas encompass a rare group of heterogeneous neoplasms that arise in bone and soft tissues in 


\section{A. CNV Comparison on Chromosome 1}

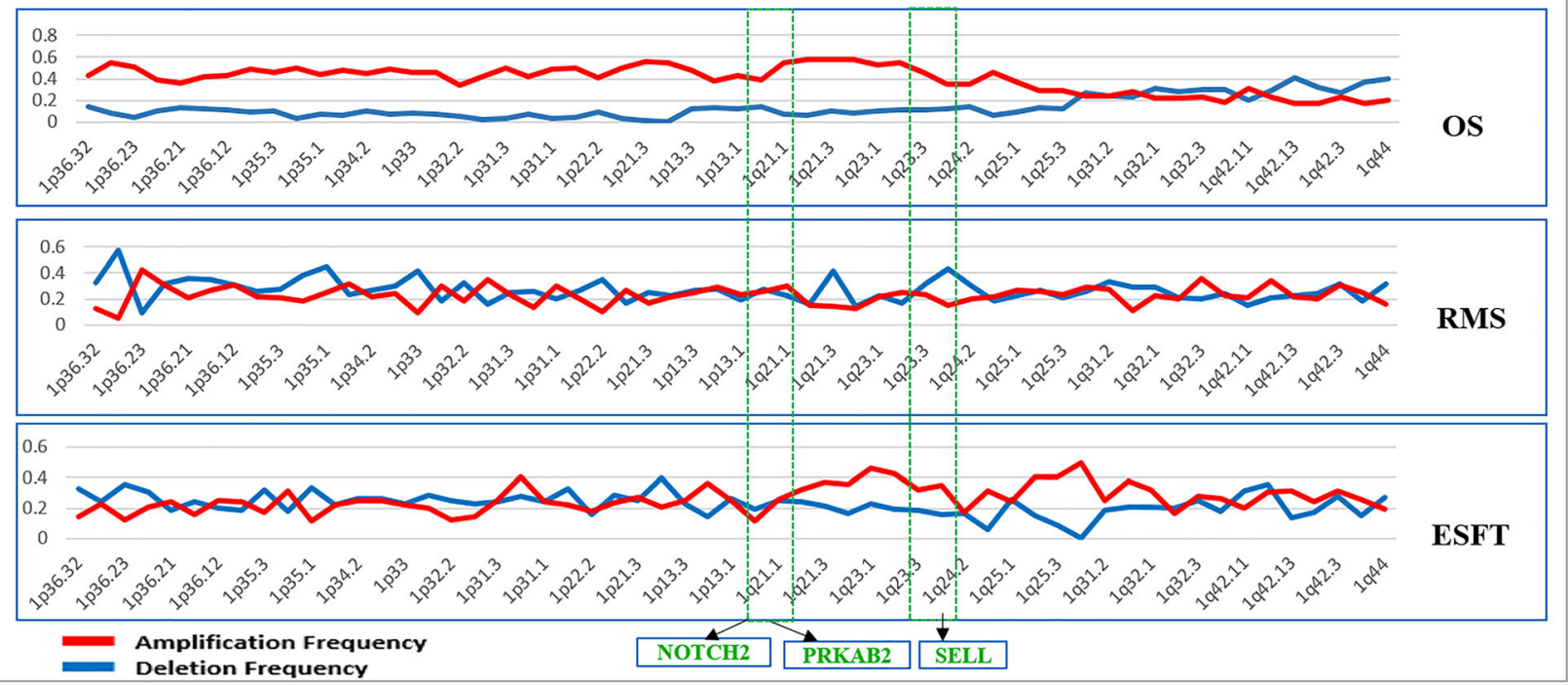

\section{B. CNV Comparison on Chromosome 8}

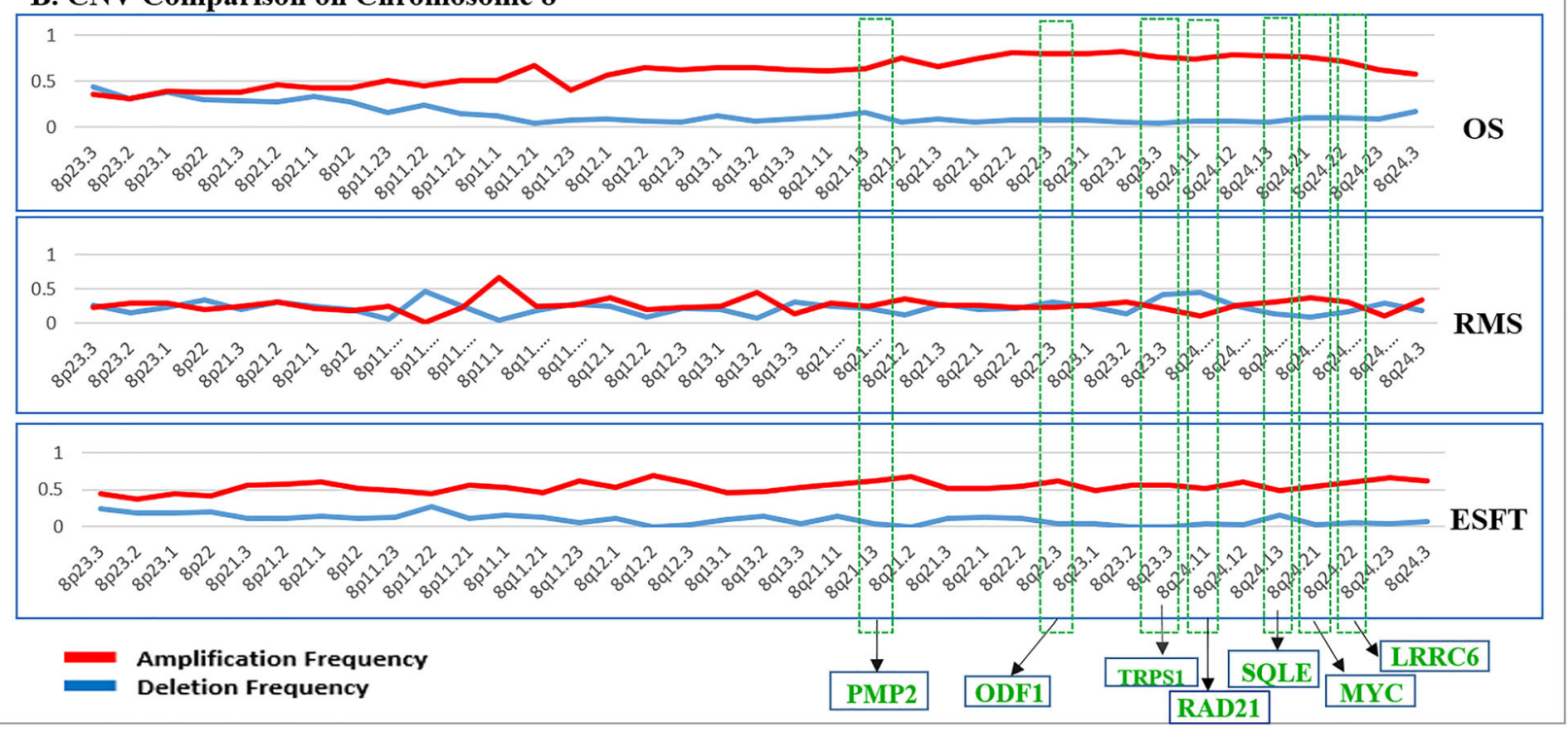

Fig. 4 Comparison of CNVs in chromosomes 1 and 8 in OS, RMS, and ESFT patient tumors. a CNVs present on chromosome 1 among pediatric sarcomas (OS, RMS, and ESFTs). b Variation of amplification and deletion frequencies across different band regions in chromosome 8 . Several genes associated with sarcoma progression are highlighted in green. Red denotes the amplification frequency while blue represents the deletion frequency

the body [1]. Despite the multi-modality approach for treating pediatric sarcomas, clinical outcomes for these patients still remains relatively poor due to onset of relapse/recurrence initiated by various molecular alterations [8-10]. While certain pediatric sarcomas like RMS and ESFTs are more genetically defined by having chromosomal translocations, other pediatric sarcomas such as OS are considered to be more genetically complex in nature [23-26]. For instance, ESFTs are genetically characterized by specific chromosomal translocations $\mathrm{t}(11 ; 22)$ (q24;q12) in $85 \%$ of ESFTs [28]. However, the remaining $15 \%$ of ESFTs have other chromosomal translocations, which involve other members of the FET and ETS family [9]. Similarly, alveolar rhabdomyosarcoma is characterized by a chromosomal translocation $\mathrm{t}(2 ; 13)$ (q35;q14) or $\mathrm{t}(1 ; 13)(\mathrm{p} 36 ; \mathrm{q} 14)$ fusing the PAX3 or PAX7 with FOXO1 $[17,18]$. On the contrary, in sporadic osteosarcoma there are various genetic alterations such as aberrations on chromosomes $15 \mathrm{q}$ and $8 \mathrm{p}$ where inconsistent rearrangements and copy number alteration have been observed [35-37].

Regardless of their genetic landscape, efforts by several multi-institutional groups have been on-going to investigate 
Table 3 CNVs (amplifications and/or deletions) frequently found in the 63 genes that serve as prognostic biomarkers for pediatric sarcomas (OS, RMS, and ESFTS)

\begin{tabular}{|c|c|c|c|c|c|c|c|c|c|c|c|}
\hline \multirow[b]{2}{*}{$\mathrm{NO}$} & \multirow[b]{2}{*}{ Band } & \multirow[b]{2}{*}{ Gene } & \multicolumn{2}{|c|}{ OS frequency } & \multicolumn{2}{|c|}{ RMS frequency } & \multicolumn{2}{|c|}{ ESFT frequency } & \multicolumn{3}{|c|}{ Sarcoma types $($ Yes $=1)$} \\
\hline & & & Amp & Del & Amp & Del & Amp & Del & $\overline{O S}$ & RMS & ESFT \\
\hline 1 & $7 p 11.2$ & $\mathrm{EGFR}^{\mathrm{a}}$ & 0.250 & 0.094 & 0.087 & 0.109 & 0.438 & 0.000 & 1 & 1 & 1 \\
\hline 2 & $12 q 13.3$ & GLI1 & 0.156 & 0.375 & 0.891 & 0.000 & 0.313 & 0.188 & & 1 & 1 \\
\hline 3 & $2 q 36.1$ & PAX3 & 0.063 & 0.531 & 0.413 & 0.000 & 0.250 & 0.063 & & 1 & 1 \\
\hline 4 & $7 q 36.1$ & $\mathrm{EZH} 2^{\mathrm{a}}$ & 0.344 & 0.094 & 0.413 & 0.000 & 0.313 & 0.125 & & 1 & 1 \\
\hline 5 & $8 q 24.21$ & MYC & 0.781 & 0.031 & 0.043 & 0.326 & 0.625 & 0.000 & 1 & 1 & \\
\hline 6 & $8 q 24.22$ & LRRC6 & 0.625 & 0.031 & 0.391 & 0.043 & 0.625 & 0.063 & 1 & 1 & \\
\hline 7 & $8 q 24.13$ & MTSS1 & 0.625 & 0.063 & 0.283 & 0.000 & 0.438 & 0.000 & 1 & 1 & \\
\hline 8 & $15 q 11.2$ & MKRN3 & 0.219 & 0.188 & 0.065 & 0.109 & 0.438 & 0.125 & 1 & 1 & \\
\hline 9 & $11 \mathrm{q} 24.2$ & ST3GAL4 & 0.156 & 0.281 & 0.174 & 0.130 & 0.438 & 0.125 & 1 & 1 & \\
\hline 10 & $15 q 22.2$ & TPM1 & 0.094 & 0.156 & 0.000 & 0.261 & 0.125 & 0.063 & 1 & 1 & \\
\hline 11 & $11 \mathrm{q} 23.1$ & IL18 & 0.063 & 0.375 & 0.043 & 0.283 & 0.000 & 0.500 & 1 & 1 & \\
\hline 12 & $11 p 15.4$ & TRIM21 & 0.063 & 0.500 & 0.174 & 0.109 & 0.000 & 0.563 & 1 & 1 & \\
\hline 13 & $10 q 23.31$ & ACTA2 & 0.000 & 0.906 & 0.087 & 0.326 & 0.125 & 0.375 & 1 & 1 & \\
\hline 14 & $8 q 22.3$ & ODF1 & 0.875 & 0.031 & 0.413 & 0.087 & 0.688 & 0.188 & 1 & & \\
\hline 15 & $8 q 24.13$ & SQLE & 0.875 & 0.031 & 0.696 & 0.022 & 0.938 & 0.063 & 1 & & \\
\hline 16 & $8 q 24.11$ & RAD21 & 0.781 & 0.031 & 0.304 & 0.022 & 0.625 & 0.063 & 1 & & \\
\hline 17 & $8 q 23.3$ & TRPS1 & 0.656 & 0.063 & 0.283 & 0.000 & 0.500 & 0.063 & 1 & & \\
\hline 18 & $8 q 21.13$ & PMP2 & 0.594 & 0.094 & 0.913 & 0.000 & 0.125 & 0.375 & 1 & & \\
\hline 19 & $8 q 24.13$ & TMEM65 & 0.531 & 0.063 & 0.217 & 0.087 & 0.500 & 0.188 & 1 & & \\
\hline 20 & $1 \mathrm{q} 24.2$ & SELL & 0.688 & 0.063 & 0.674 & 0.000 & 0.188 & 0.125 & 1 & & \\
\hline 21 & $15 q 26.3$ & SNRPA1 & 0.688 & 0.125 & 0.000 & 1.000 & 0.125 & 0.188 & 1 & & \\
\hline 22 & $22 q 11.21$ & MAPK $1^{a}$ & 0.406 & 0.219 & 0.000 & 0.587 & 0.000 & 0.688 & 1 & & \\
\hline 23 & $15 q 26.3$ & IGF1R & 0.313 & 0.094 & 0.130 & 0.065 & 0.125 & 0.000 & 1 & & \\
\hline 24 & $15 q 26.1$ & KIF7 & 0.563 & 0.063 & 0.065 & 0.435 & 0.375 & 0.000 & 1 & & \\
\hline 25 & $12 \mathrm{p} 13.31$ & CD163 & 0.500 & 0.063 & 0.174 & 0.152 & 0.125 & 0.125 & 1 & & \\
\hline 26 & $16 p 13.3$ & MAPK8IP3 & 0.000 & 0.813 & 0.630 & 0.043 & 0.688 & 0.000 & 1 & & \\
\hline 27 & $10 q 22.1$ & NUDT13 & 0.000 & 0.781 & 0.739 & 0.000 & 0.125 & 0.438 & 1 & & \\
\hline 28 & $10 q 22.1$ & $\mathrm{P} 4 \mathrm{HA} 1$ & 0.000 & 0.688 & 0.935 & 0.000 & 0.125 & 0.313 & 1 & & \\
\hline 29 & $10 q 23.1$ & TSPAN14 & 0.000 & 0.688 & 0.043 & 0.413 & 0.000 & 0.750 & 1 & & \\
\hline 30 & $11 q 13.1$ & CFL1 & 0.406 & 0.125 & 1.000 & 0.000 & 0.063 & 0.313 & & 1 & \\
\hline 31 & $9 q 22.33$ & ALG2 & 0.625 & 0.031 & 1.000 & 0.000 & 0.500 & 0.000 & & 1 & \\
\hline 32 & $1 \mathrm{q} 21.1$ & PRKAB2 & 0.406 & 0.063 & 1.000 & 0.000 & 0.313 & 0.188 & & 1 & \\
\hline 33 & $16 p 11.2$ & ITGAL & 0.063 & 0.375 & 0.978 & 0.000 & 0.375 & 0.250 & & 1 & \\
\hline 34 & $7 q 21.2$ & PEX1 & 0.438 & 0.094 & 0.978 & 0.022 & 0.063 & 0.438 & & 1 & \\
\hline 35 & $3 p 21.1$ & $\mathrm{PRKCD}^{\mathrm{a}}$ & 0.125 & 0.156 & 0.978 & 0.000 & 0.063 & 0.250 & & 1 & \\
\hline 36 & $12 q 21.1$ & THAP2 & 0.344 & 0.219 & 0.978 & 0.000 & 0.438 & 0.063 & & 1 & \\
\hline 37 & $19 q 13.33$ & $\mathrm{AP} 2 \mathrm{~A} 1$ & 0.125 & 0.063 & 0.935 & 0.000 & 0.063 & 0.375 & & 1 & \\
\hline 38 & $16 p 11.2$ & ITGAM & 0.031 & 0.406 & 0.913 & 0.000 & 0.063 & 0.250 & & 1 & \\
\hline 39 & 10p14 & $\mathrm{KIN}$ & 0.031 & 0.563 & 0.913 & 0.000 & 0.125 & 0.375 & & 1 & \\
\hline 41 & $12 q 15$ & MDM2 & 0.156 & 0.187 & 0.391 & 0.130 & 0.125 & 0.500 & & 1 & \\
\hline 40 & $11 p 15.5$ & $\mid G F 2^{a}$ & 0.1875 & 0.1875 & 0.283 & 0.0217 & 0.75 & 0 & & 1 & \\
\hline 42 & $11 q 25$ & B3GAT1 & 0.063 & 0.125 & 0.000 & 1.000 & 0.813 & 0.000 & & 1 & \\
\hline
\end{tabular}


Table 3 CNVs (amplifications and/or deletions) frequently found in the 63 genes that serve as prognostic biomarkers for pediatric sarcomas (OS, RMS, and ESFTS) (Continued)

\begin{tabular}{|c|c|c|c|c|c|c|c|c|c|c|c|}
\hline \multirow[b]{2}{*}{$\mathrm{NO}$} & \multirow[b]{2}{*}{ Band } & \multirow[b]{2}{*}{ Gene } & \multicolumn{2}{|c|}{ OS frequency } & \multicolumn{2}{|c|}{ RMS frequency } & \multicolumn{2}{|c|}{$\underline{\text { ESFT frequency }}$} & \multicolumn{3}{|c|}{$\underline{\text { Sarcoma types }(Y e s=1)}$} \\
\hline & & & Amp & Del & Amp & Del & Amp & Del & OS & RMS & ESFT \\
\hline 43 & $11 q 13.1$ & PYGM & 0.563 & 0.063 & 0.000 & 1.000 & 0.313 & 0.063 & & 1 & \\
\hline 44 & $11 q 13.1$ & RELA & 0.031 & 0.688 & 0.000 & 1.000 & 0.625 & 0.063 & & 1 & \\
\hline 45 & $3 p 14.1$ & PSMD6 & 0.125 & 0.656 & 0.000 & 1.000 & 0.250 & 0.000 & & 1 & \\
\hline 46 & $1 \mathrm{p} 12$ & $\mathrm{NOTCH} 2$ & 0.500 & 0.000 & 0.000 & 1.000 & 0.375 & 0.125 & & 1 & \\
\hline 47 & $12 \mathrm{p} 13.2$ & ETV6 & 0.156 & 0.156 & 0.239 & 0.000 & 0.750 & 0.000 & & & 1 \\
\hline 48 & $5 q 32$ & PDGFRB $^{a}$ & 0.063 & 0.531 & 0.065 & 0.870 & 0.750 & 0.000 & & & 1 \\
\hline 49 & $7 p 12.3$ & IGFBP3 & 0.156 & 0.188 & 0.065 & 0.000 & 0.688 & 0.000 & & & 1 \\
\hline 50 & $7 p 21.2$ & ETV1 & 0.156 & 0.063 & 0.152 & 0.000 & 0.563 & 0.000 & & & 1 \\
\hline 51 & $7 q 33$ & CREB3L2 & 0.281 & 0.094 & 0.087 & 0.152 & 0.125 & 0.000 & & & 1 \\
\hline 52 & $17 q 21.31$ & ETV4 & 0.094 & 0.250 & 0.196 & 0.196 & 0.563 & 0.063 & & & 1 \\
\hline 53 & 10p11.21 & ANKRD30A & 0.094 & 0.438 & 0.196 & 0.000 & 0.500 & 0.125 & & & 1 \\
\hline 54 & $4 q 12$ & $\mathrm{KIT}^{\mathrm{a}}$ & 0.219 & 0.063 & 0.000 & 0.130 & 0.375 & 0.000 & & & 1 \\
\hline 55 & $12 q 23.2$ & $|G F|^{a}$ & 0.125 & 0.094 & 0.130 & 0.043 & 0.250 & 0.000 & & & 1 \\
\hline 56 & $21 q 22.2$ & ERG & 0.469 & 0.063 & 0.109 & 0.022 & 0.188 & 0.063 & & & 1 \\
\hline 57 & $20 q 13.2$ & NFATC2 & 0.313 & 0.219 & 0.000 & 0.326 & 0.188 & 0.063 & & & 1 \\
\hline 58 & $11 q 24.3$ & FLI1 & 0.156 & 0.125 & 0.043 & 0.348 & 0.188 & 0.125 & & & 1 \\
\hline 59 & $x q 25$ & STAG2 & 0.031 & 0.813 & 0.326 & 0.022 & 0.125 & 0.750 & & & 1 \\
\hline 60 & xp11.4 & BCOR & 0.000 & 0.938 & 0.130 & 0.217 & 0.438 & 0.563 & & & 1 \\
\hline 61 & $17 q 12$ & TAF15 & 0.188 & 0.313 & 0.370 & 0.022 & 0.125 & 0.500 & & & 1 \\
\hline 62 & $6 \mathrm{p} 22.3$ & KIAA0319 & 0.375 & 0.031 & 0.022 & 0.391 & 0.063 & 0.500 & & & 1 \\
\hline 63 & $12 q 13.12$ & ATF1 & 0.281 & 0.125 & 0.000 & 0.326 & 0.313 & 0.438 & & & 1 \\
\hline
\end{tabular}

Note: ${ }^{a}$ denotes gene with druggable targets by DrugBank annotation. Designation of "1" indicates there is literature to support that specific gene is deleted or amplified for that sarcoma type. All frequency calculation is based on 206 sarcoma patients CNVs

novel therapeutic options for improving overall survival for these pediatric malignancies. However, even with these advancements, the 5-year survival rates for relapsed/recurrent pediatric sarcoma patients still remain less than $30 \%[1,2]$. Therefore, along with identifying downstream targets of these molecularly-characterized and complex pediatric sarcomas, it is equally imperative to assess and identify other acquired genetic changes such as CNVs involving genetic amplifications and/or deletions that may provide novel therapeutic options to improve clinical outcomes [29]. Notably, OS, RMS, and ESFT exhibit various CNVs that can serve as prognostic biomarkers for these pediatric sarcomas [23-31]. Our objective for this study was to identify CNVs common to all three of the pediatric sarcomas (OS, RMS, ESFTs) and evaluate the role of these CNVs in response to DNA damaging agents to determine if they are predictive biomarkers of therapeutic response. This comprehensive study investigated band and gene alterations of somatic copy number amplification and deletion in 27 bone and soft tissue sarcoma using aCGH arrays (Affymetrix). Due to increased availability of publicly available datasets, improved and efficient resources for integrative genomic sequencing, and molecular characterization of patient-specific tumors it is now feasible and could be potentially used to guide selection of personalized therapies.

Through our comparative genomic analyses of OS, RMS, and ESFTs and healthy subjects, we identified CNVs (amplifications and deletions) in various chromosomal regions (Fig. 2). Bioinformatics analyses was also conducted to identify the pattern of genomic instability in these pediatric sarcomas. To the best of our knowledge, this is the first study to compare genomic instabilities between OS, RMS, ESFTs and healthy population controls. Genes associated with survival and/ recurrence of these sarcomas with statistical significance were found on long arm of chromosome 8 with much higher amplification frequency observed in OS $(0.8-0.92)$. These include MYC (8q24.21), LRRC6 (8q24.22), MTSS1 (8q24.13), ODF1 (8q22.3), SQLE (8q24.13), RAD21 (8q24.11), TRPS1 (8q23.2), PMP2 (8q21.13), TMEM65 (8424.13). In ESFTs, there is higher amplification frequency $(0.5-0.7)$ for majority of the bands and lower deletion frequency (00.1 ) in chromosome 8. Similar results are obtained in RMS. CNVs, in particular, amplifications involving chromosome 8 have also been reported by other groups in OS, RMS, and ESFTs, thus, further validating our 


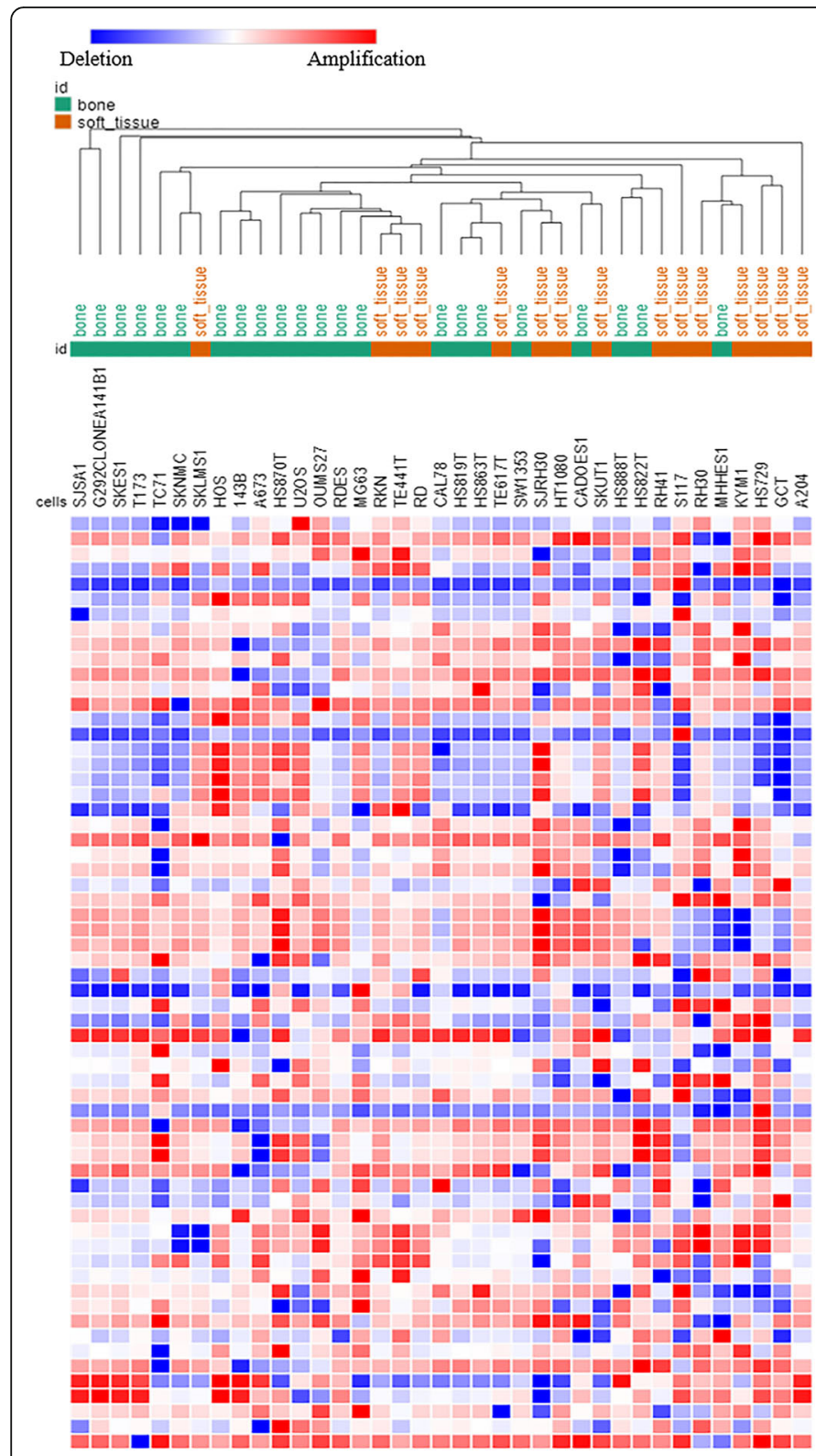

Sarcoma cell lines

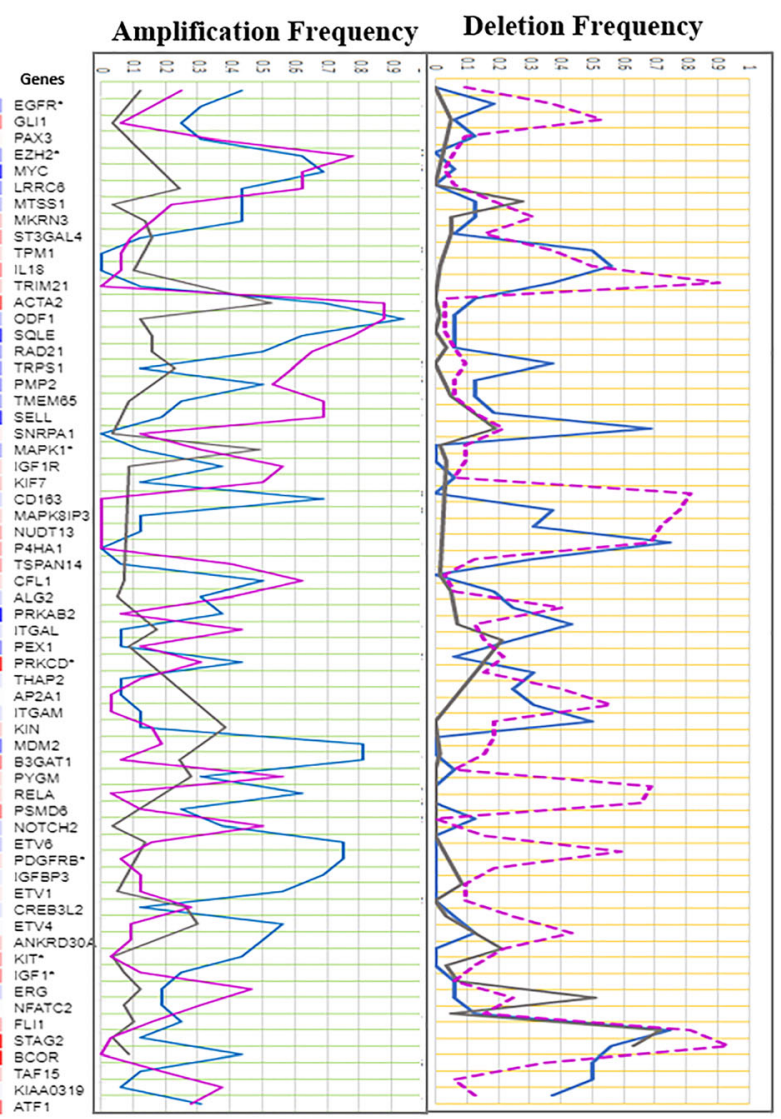

Sarcoma patients

Fig. 5 Comparison of CNVs between sarcoma patients and sarcoma cell lines. The left panel represents the CNVs on 27 sarcoma cells, where green is type of bone sarcoma and brown is type of soft tissue sarcoma. The right panel represents the amplification or the deletion frequency of copy number variation in sarcoma patients, which is associated with CNVs of the sarcoma cells

data [23-31]. While further exploration is needed to assess the role and function of many of the amplified genes present on chromosome 8 in pediatric sarcomas, one key gene that has been highly studied in these pediatric sarcomas is MYC, which has a role in various other cancers [36, 37]. MYC is a transcription factor that is known to regulate critical biological functions such as cell cycle, apoptosis, and metabolism [36]. Genetic alterations that result in changes to MYC, such as MYC amplification, can dysregulate its normal function and alter the balance between being a tumor suppressor versus being tumorigenic [36]. Along with chromosomal changes observed in chromosome 8 , smaller aberrations in OS, RMS, and ESFTs were also identified at chromosomes 1q, 12q and $\mathrm{x}$. The long arm (1q) of chromosome 1 also signifies amplification with gene SELL showing higher significance in OS. The majority of the bands in the long arm (1q) of chromosome 1 have an amplification frequency $0.2-0.4$ while the deletion frequency is between $0.1-0.2$ in ES. Several CNV 


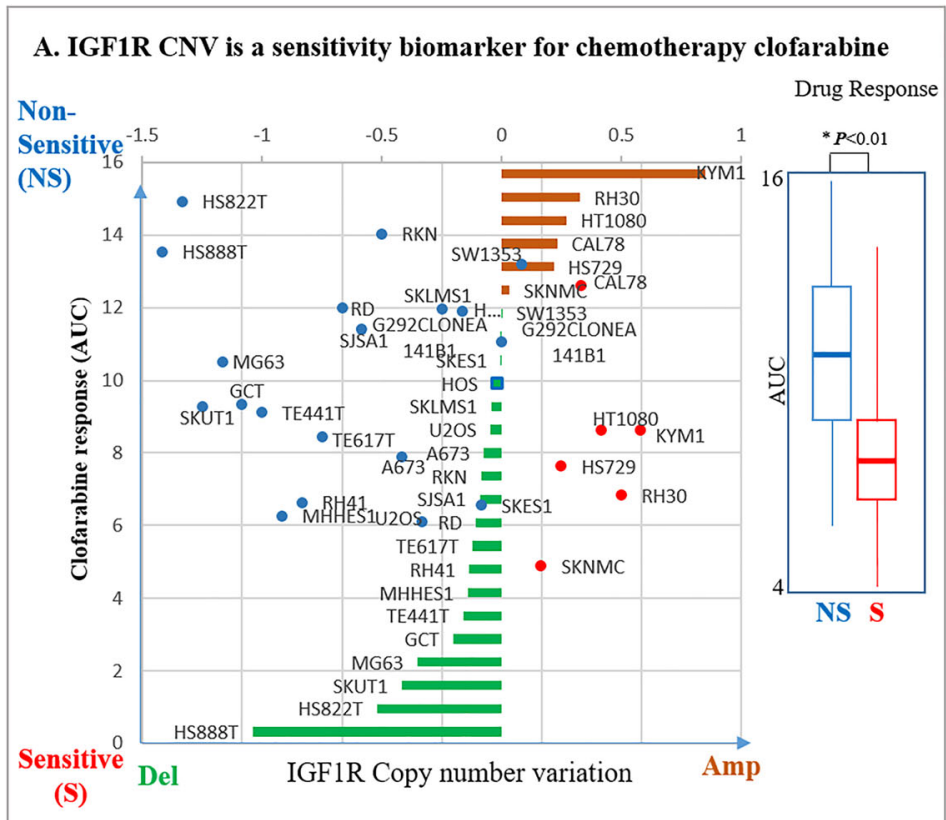

\section{B. Strong linear correlation IGF1R CNV with clofarabine response}

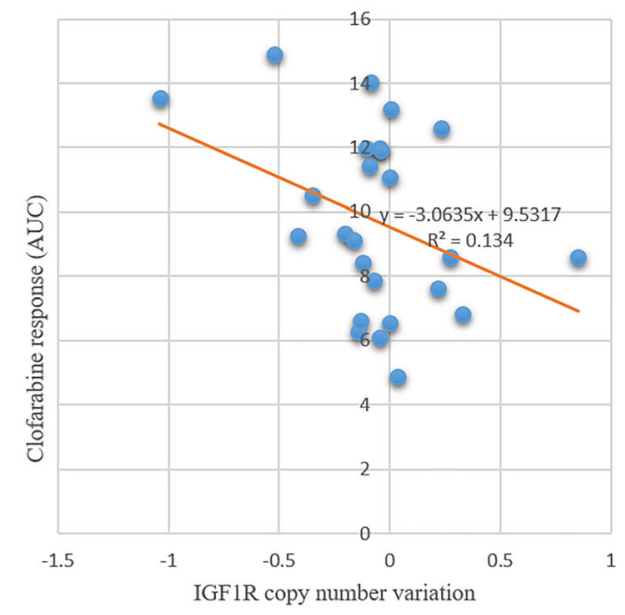

$r($ Clofarabine response, IGF1R CNV $)=-0.3661$

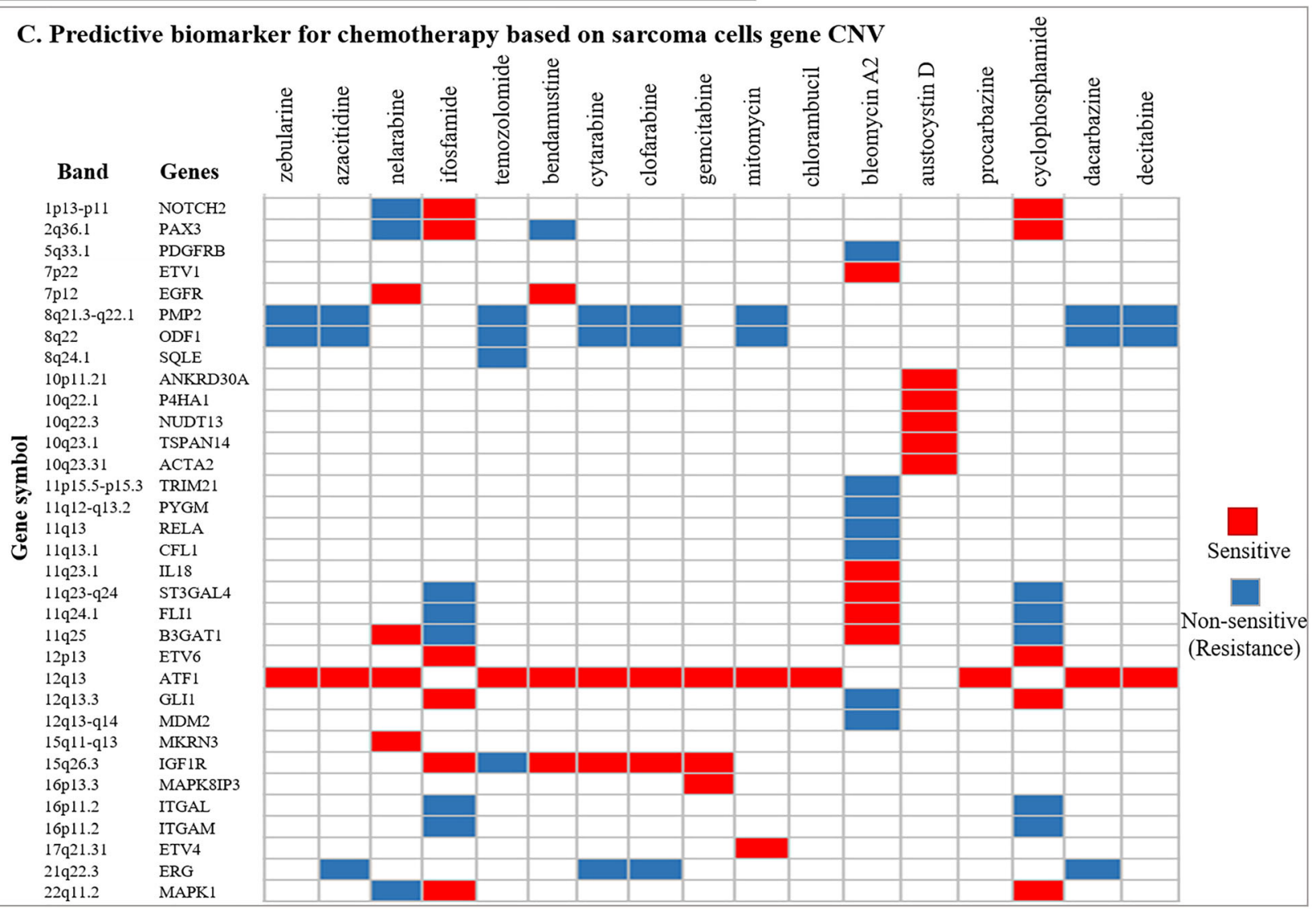

Fig. 6 (See legend on next page.) 
(See figure on previous page.)

Fig. 6 CNVs as predictive biomarker for chemotherapy in pediatric sarcoma. Gene IGF1R and clofarabine response provide an example of data integration. IGF1R gene amplification correlates with increasing sensitivity to clofarabine in pediatric sarcoma. a Correlation of IGF1R CNV and relative sensitivity to clofarabine. The IGF1R CNV status (deletion or amplification) for each cell line is presented in the middle of the panel (Del = gene deletion denoted in green; $A m p=$ gene amplification denoted in red). The $y$ axis signifies the response of each cell line to clofarabine and is presented as the area under the curve (AUC) to drug response. Blue circles = cell lines with IGF1R deletion; Red circles = cell lines with IGF1R amplification (left panel); Drug response data compiled as non-sensitive (NS) versus sensitive (S) cell lines, ${ }^{*} p<0.01$, IGF1R deletion vs. IGF1R amplification, right panel b Strong linear correlation between IGF1R CNV and clofarabine response. Blue circles = cellular response to clofarabine; $y$-axis = AUC and x-axis = CNV of IGF1R. (c) CNVs as chemotherapy biomarkers in sarcoma. The sensitive and non-sensitive biomarker selection is based on the threshold where a threshold of $p<0.05$ and correlation coefficient $r>0.35$ denotes non-sensitivity and $r<-0.35$ signifies sensitivity

analyses $[17,28,30,31]$ have validated and verified the accuracy of our results.

However, CNVs associated with recurrence in these pediatric sarcomas correlate with poor prognosis by specific chromosomal translocations or variations in OS, RMS, and ESFTs that can serve as prognostic biomarkers for these diseases [4-7]. To date, the correlation between these prognostic biomarkers and their response to therapies still requires further exploration using in vivo pediatric sarcoma models.

We identified CNVs in 63 genes among the three pediatric sarcomas (OS, RMS, and ESFTs) that correlated with the recurrence of the diseases, suggesting CNVs in the 63 genes may provide prognostic biomarkers for these sarcomas. The 63 genes have high frequency of amplifications as well as deletions in these sarcomas. For example, genes such as KIF7, IGF1R and SNRPA1 on 15q16.1-15q16.4 are amplified in OS. In RMS amplification of PAX3 (2q36.1) with frequency of 0.413 was observed, whereas, a high deletion frequency of 0.9-1 was evident in CFL1, ALG2, PRKAB2, ITGAL, PEX1, PRKCD, AP2A1, KIN, ITGAM, THAP2 genes. ESFTs exhibit frequently mutated STAG2 on chromosome Xq25 [2, 40] with a high deletion frequency of 0.75 in our study.

By integrating large-scale drug screening to evaluate drug response profiles of the CNVs identified in 63 genes from 27 sarcoma cell lines it was identified that 33 genes with CNVs had either sensitive or non-sensitive responses to 17 chemotherapies. The CNVs in these 33 genes could serve as potential predictive biomarkers of therapeutic response which still needs to be further explored. An example of this included the CNVs identified in IGFR1 (Table 3). IGFR1 is receptor for the growth hormone insulin growth factor (IGF) which can mediate cell proliferation [26]. Binding of IGF to IGFR1 initiates downstream singling cascades to increase cell proliferation and decrease apoptosis, which is observed in these pediatric sarcomas [26]. Figure 6a, b show that CNVs in IGFR1 result in IGFR1 serving as a sensitive biomarker of therapeutic response to Clofarabine. Clofarabine is a purine nucleoside analog that can inhibit DNA/RNA polymerases and promotes apoptosis of cancer cells [41, 42]. This study provides novel insights into how genetic alterations such as CNVs can potentially serve as both prognostic biomarkers and predictive biomarkers of therapeutic response in pediatric sarcomas. The systems pharmacology approach described here provides a platform to personalize therapies that have could improve clinical outcomes in aggressive pediatric malignancies $[43,44]$.

\section{Conclusions}

In our study, we evaluated CNVs as well as their frequencies of amplification (copy number gain) and deletion (copy number loss) in a large cohort of OS, ESFTs, and RMS patient samples and sarcoma cell lines. To the best of our knowledge, this is the first study screening genomic-profiling (CNVs) of aggressive pediatric sarcomas and assessing their drug-responses to potentially improve therapeutic and clinical outcomes in these aggressive diseases. Our future studies will be focused on functionally validating identified targets using in vivo modeling approaches and evaluating their roles as a potential predictive and/or prognostic biomarker in our quest to improve the currently dismal therapeutic outcomes in pediatric sarcoma patients.

\section{Methods}

\section{Data collection}

\section{Healthy subjects}

The comprehensive genomic structure variation data for the healthy individuals was collected from the Database of Genomic Variants [20]. Fifty-five published studies were included in DGV, from the well-known archival SV databases including, dbVar (NCBI) and DGVa (EBI). The latest dataset GRCh 37 (hg19) version released on May 15, 2016 is collected [45]. A total of 488,630 variant records in 22,255 samples were used to study the CNVs representing a total of 14,316 non-redundant individuals across $\sim 44$ different populations representing both males and females almost equally. Each of these entities contain multiple studies from different analysis. Insertion, deletion, duplication, tandem duplication, novel sequence insertion and mobile element insertion in chromosomes were investigated. All genomic variants in DGV were detected by different experiment methods, including Bacterial Artificial Chromosome (BAC) and oligonucleotide-based chromosomal Comparative Genomic Hybridization (Oligo-cCGH), aCGH, fluorescence in situ hybridization (FISH), 
polymerase chain reaction (PCR), sequencing, single nucleotide polymorphism (SNP) array and Digital array. The latest data consists of $44 \%$ from microarray studies, $33 \%$ from sequencing and 3\% from FISH/PCR and Optimal Mapping. The size of the DNA segment for $\mathrm{CNV}$ ranges from $50 \mathrm{bp}$ to $3 \mathrm{Mb}$, with lesser number of variants in the range of $50 \mathrm{bp}$ to $1 \mathrm{~Kb}$ range. This is because the majority of the CNV detected using microarray is large-scale CNV. All genome region segments of CNVs were obtained and mapped to genes and bands for further study.

\section{OS}

One hundred seventeen OS are collected from TARGET and GEO database respectively, where both data sets were tested by Affymetrix Genome-Wide Human SNP 6.0 Array chips (GEO platform accession ID, GPL6801). 85 samples from TARGET [12] were obtained and segments of CNVs with level 3 data were selected. $32 \mathrm{CEL}$ files of $\mathrm{CNV}$ profile were obtained from $\mathrm{GEO}$ accession ID, GSE33383 with high-grade OSs. Both datasets provide clinical information about each subject including recruitment, demographics, survival and physical examinations (Table 4).

\section{ESFTs}

CGH profiling of 25 ESFT tumor samples, from GEO accession ID, GSE8398 (65), were scanned on Agilent-013282 Human Genome CGH Microarray 44B (GEO platform accession ID, GPL2879). All 25 sample CEL files were used for data analysis. The datasets provided detailed clinical information of samples, such as disease stage, site of disease, occurrence of metastasis and patient status (Table 4).

\section{RMS}

CGH profilings of 64 alveolar RMS genome variation, from GEO accession ID, GSE24715. 7 sample genome variation was tested by Affymetrix Mapping $250 \mathrm{~K}$ Sty2 SNP chips (GPL3720 in GEO, 238378 probe sets), while 57 samples were tested on Affymetrix Human Mapping $50 \mathrm{~K}$ Xba240 SNP Array (GEO platform ID: GPL2005). The raw CEL files were used to generate the CNVs, which were further analyzed for deletion and amplification frequencies.

\section{Cancer cell line encyclopedia}

All segments of copy number variations for 27 sarcoma cell lines were collected from CCLE, which were tested by Affymetrix Genome-Wide Human SNP Array 6.0. Log 2 transformed segment values were used for further analysis.

\section{Data pre-processing and gene annotation}

All CEL files obtained from GEO were quantified using the Bioconductor package in R. The Oligo library was used to obtain the copy number values for the DNA segments by MAS 5.0 algorithm. The normalized $\log _{2}$ ratio (healthy/tumor) on probe-sets was annotated to genes for further analysis. All continuous variable CNV will be

Table 4 Demographics and clinical characteristics of sarcoma patients

\begin{tabular}{|c|c|c|c|c|}
\hline Sarcoma Type & Data Source & & Clinical Information & Number of Samples \\
\hline \multirow[t]{9}{*}{ OS } & \multirow[t]{9}{*}{ TARGET } & \multirow[t]{2}{*}{ Stage1 } & Relapse & 41 \\
\hline & & & Non-Relapse & 44 \\
\hline & & \multirow[t]{2}{*}{ Stage2 } & Metastatic & 22 \\
\hline & & & Non-Metastatic & 63 \\
\hline & & \multirow[t]{2}{*}{ Gender } & Female & 37 \\
\hline & & & Male & 48 \\
\hline & & \multirow[t]{2}{*}{ Vital Status } & Alive & 55 \\
\hline & & & Dead & 30 \\
\hline & & Age & $\begin{array}{l}\text { At the time of Diagnosis (days): } \\
\text { 1299-11,828 }\end{array}$ & 85 \\
\hline High Grade OS & GEO-GSE33383 & Age & $\begin{array}{l}\text { At the time of Diagnosis (days): } \\
3072-14,965\end{array}$ & 32 \\
\hline \multirow[t]{6}{*}{ ESFTS } & \multirow[t]{6}{*}{ GEO-GSE8398 } & \multirow[t]{3}{*}{ Gender } & Female & 11 \\
\hline & & & Male & 9 \\
\hline & & & Unknown & 5 \\
\hline & & \multirow[t]{3}{*}{ Stage } & Metastatic & 11 \\
\hline & & & Localized & 9 \\
\hline & & & Unknown & 5 \\
\hline RMS & GEO-GSE24715 & Histology Subtype & ARMS (Alveolar rhabdomyosarcoma) & 64 \\
\hline
\end{tabular}


changed into five level value, where -2 is significant deletion, -1 is deletion, 0 is no change of CNV, 1 is amplification and 2 is significant amplification. Base on whole $\mathrm{CNV}$ histogram distribution of a particular array platform, the CNV value is larger than top 5\% range, we set $\mathrm{CNV}$ as 2; when the CNV is large than $15 \%$ range and less than top $5 \%$ range, it sets as 1 . The $\mathrm{CNV}$ value is less than negative $5 \%$ range; we set $\mathrm{CNV}$ as -2 . The $\mathrm{CNV}$ value is less than negative $15 \%$ range and larger than negative $5 \%$ range, it sets -1 , others sets 0 .

The HGNC (HUGO Gene Nomenclature Committee, http://www.genenames.org/) database provides researchers with standard gene names for the human genome to avoid the complexity of multiple overlapping and conflicting nomenclature systems. The database currently consists of around 24,000 genes and their corresponding approved gene symbols. Each gene has a unique HGNC ID which makes it easier to identify the gene type. Genes were also annotated with other information including gene synonyms, uniprot ids, refseq ids, previous gene symbols and a functional description about each gene, all of which aids in integrating the information from the NCBI or other databases [46].

By software Bedtools 'intersectBed', we mapped genome region segments of CNV to gene symbols by GRCh37/ hg19 genome annotation file [38]. All segment data records were changed into individual genes and associated bands on chromosomes. For this work, all the genes were mapped to their standard HGNC name using the annotations from Ensemble-Biomart [45] for multi-data integration and comparison.

\section{High-resolution array comparative genomic hybridization (aCGH) Chip and Assay}

(1) Affymetrix Genome-Wide Human SNP 6.0 Array (GPL6801) contains 934,946 SNPs and 946,371 non-polymorphic probes for the detection of CNVs. Enzymes Nsp I and Sty I were used in parallel in the assay to digest and fragment DNA. Probes on the SNP Array 6.0 are targeting sequences that may sit on fragments cut by either enzymes or both. All SNP probes occur in a Nsp, Sty or Nsp + Sty fragment, but the $\mathrm{CN}$ probes occur only Nsp and Nsp + Sty fragments (not Sty-alone fragments). The total genomic DNA (500 ng) was digested with Nsp I and Sty I restriction enzymes into fragments and ligated to adaptors that recognize the cohesive $4 \mathrm{bp}$ overhangs. A generic primer that recognizes the adaptor sequence was used to amplify adaptorligated DNA fragments. The amplified DNA is then labeled and hybridized to a SNP Array 6.0. PCR conditions will be optimized to preferentially amplify fragments in the 200 to $1100 \mathrm{bp}$ size range.
The Birdsuite software is applied here to identify rare $\mathrm{CNVs}$ from the Affymetrix SNP 6.0 array via a onedimensional Gaussian mixture model (GMM) [39]. We matched $946,371 \mathrm{CNV}$ probes to 22,891 genes by GEO released platform GPL6801 annotation.

(2) The GeneChip ${ }^{\circ}$ Human Mapping $500 \mathrm{~K}$ Array is one of the aCGH chips designed by Affymetrix Company. It is comprised of two arrays, each capable of genotyping on average 250,000 SNPs (approximately 262,000 for Nsp arrays corresponding to CNV and 238,000 for Sty arrays associated with SNPs. GPL3720 in GEO is a platform of Affymetrix Mapping 250 K Sty2 SNP Array, which is a subset of the GeneChip Human Mapping $500 \mathrm{~K}$ Array Set. The array has probes for CNVs and each marker can be interrogated with up to five probes, ensuring cross-verification for data integrity [http://www.affymetrix.com/support/ technical/byproduct.affx?product $=500 \mathrm{k}]$.

Affymetrix Human Mapping $50 \mathrm{~K}$ Xba240 SNP Array is the GeneChip Mapping $100 \mathrm{~K}$ Set for SNPs (GPL2005). It is comprised of a set of two arrays that enable genotyping of greater than 100,000 SNPs with a single primer. All CEL files are normalized to digital number by Affymatrix NET 'cdf' package in R [http://www.affymetrix.com/ support/technical/byproduct.affx?product $=500 \mathrm{k}]$.

(3) Agilent-013282 Human Genome CGH Microarray 44B (GPL2879) is a high performance 60-mer oligonucleotide, allowing genome-wide survey and molecular profiling of DNA copy number changes on a single chip. It consists of 44,290 60-mer oligonucleotide probes, 7321 genes, empirically validated in multiple model systems, spanning coding and noncoding sequences with average spatial resolution of $35 \mathrm{~kb}$.

\section{Copy number amplification and deletion frequency calculation based on gene or band}

Copy number alterations were derived from aCGH chips and measured using $\log 2$ ratios of the fluorescence intensities from two channels ( $\mathrm{Cy} 3$ and $\mathrm{Cy} 5)$, one for the target sample and the other for the reference sample. For a given gene (or region), a negative $\log 2$ ratio is an indication of a loss, and a positive $\log 2$ ratio is an indication of a gain. If the $\log 2$ ratio equals zero, the target sample and the reference sample have the same copy number for that given gene (or region). However, it should be noted that different platforms can demonstrate differences in amplifications and deletions even when using the same strategy to normalize data from CEL file to digital number. To compensate for this variability in platforms, we used equal quartiles for integration of all pertinent datasets. 
The threshold for amplification and deletion decisions were based on the quantile distribution for any gene set, the extreme values present in lower threshold (less than $15 \%$ range, such as $<=-0.146768)$ and in upper threshold (more than $85 \%$ range, such as $>=0.147642$ ) are considered as significant threshold for deletion and amplification respectively. Similarly, for the band, the extreme values present in lower quartile (less than 15\% range, $<=-0.16109$ ) and in upper quartile (more than 85\% range, $>=1.7007)$ are considered as significant for deletion and amplification respectively.

For each of the genes, the total number of samples studied, total number of observed gains in those set of samples and total number of observed loss in the same set of samples is calculated. The amplification fraction and deletion fraction for each of the genes is then calculated using the formula below:

(i). Deletion frequency for each gene $=$ Total number of observed losses for a specific gene/Total number of samples for that gene.

(ii). Amplification frequency for each gene = Total number of observed gains for a specific gene/Total number of samples for that gene.

To study the amplification and deletion fraction for a unique band similar steps are applied as for these genes. The total number of genes is calculated in each band to a given chromosome. The amplification and deletion fraction for these bands is calculated using the formula below:

(i) Deletion frequency for each band = Total number of observed losses for a band/Total number of samples for a specific gene $\times$ Total number of genes for that specific band.

(ii) Amplification frequency for a band = Total number of observed gains for a band/Total number of samples for a specific gene $\times$ Total number of genes for that specific band.

\section{Large scale of drug screening}

Cancer Therapeutics Response Portal (CTRP v2.0) provides more than 481 small molecules screening on 664 cancer cell lines [22]. Twenty-seven sarcoma cancer cell lines were included from CTRP. Pharmacologic area under the dose-response curve test AUC is used to describe the drug response reaction. Drug efficacy estimation of AUC values, a nonparametric spline regression technique with the constraint that each drug's higher dose concentration provides at least equal or higher drug efficacy (inhibition) than its lower concentration was applied for estimating the drug activities across each drug's experimental range of dose concentration. The successive parabolic interpolation for one-dimensional optimization, implemented with the nlminb routine of $\mathrm{R}$, was used to obtain the final AUC estimates by inverting the dose-effect curves.

\section{Statistical analysis}

All code and programming were done using R. Pearson correlation coefficient is used to calculate association between drug response AUC and copy number variation in each of gene. GENE-E software (https://software.broadinstitute.org/ GENE-E/) is used for clustering analysis and visualization.

\section{Additional files}

Additional file 1: All NCCN biomarkers for sarcoma,including diagonosis and therapy determination (XLSX $17 \mathrm{~kb}$ )

Additional file 2: Samples annotation for sarcoma patients and cell lines in the study. (XLSX $45 \mathrm{~kb}$ )

Additional file 3: Comprehensive literature reviews were also conducted to collect gene CNV amplification and deletion that can be predictive of clinical outcome in three types of sarcoma from the PubMed repository. (XLSX $122 \mathrm{~kb}$ )

Additional file 4: Integrated CNVs from sarcoma cells and sarcoma patients of OS, RMS and ESFT. (XLSX 3277 kb)

\section{Abbreviations \\ aCGH: array-based $\mathrm{CGH}$; AUC: Area under the dose-response curve; BAC: Bacterial artificial chromosome; CCLE: Cancer cell line encyclopedia; CGH: Comparative genomic hybridization; CNV: Copy number variation; CTRP: Cancer therapeutics response portal; DGV: Database of genomic variants; ESFTs: Ewing's sarcoma family of tumors; FISH: Fluorescence in situ hybridization; GEO: Gene expression omnibus; HGNC: HUGO gene nomenclature committee; HUGO: Human genome organisation; LOH: The loss of heterozygosity; NCCN: National comprehensive cancer network; OligoCCGH: Oligonucleotide-based Chromosomal CGH; OS: Osteosarcoma; PCR: Polymerase chain reaction; RMS: Rhabdomyosarcoma; SNP: Single nucleotide polymorphism; TARGET: Therapeutically applicable research to generate effective treatments}

\section{Acknowledgements}

Please acknowledge Shijun Zhang who contributed reference editing and materials organization.

\section{Funding}

This work was partially supported by the National Institutes of Health grant numbers DK102694, GM10448301, LM011945, IUSCC Cancer Center Support grant P30 CA082709, and NIH/NICHD 1U54HD090215. The publication costs for this article were funded by the corresponding authors.

\section{Availability of data and materials}

The datasets analyzed during the current study are available in the DGV database [20], http://dgv.tcag.ca/dgv/app/home; CCLE [21], https://portals.broadinstitute.org/ ccle; CTRP [22] https://portals.broadinstitute.org/ctrp.v2.1/; GEO repository [https:// www.ncbi.nlm.nih.gov/sites/GDSbrowser/], https:/www.ncbi.n/m.nih.gov/geo/ and TARGET Data Matrix [https://ocg.cancer.gov/programs/target], https:// ocg.cancer.gov/programs/target/data-matrix.

All data annotation and results during this study are included in this published article and its supplementary information files, Additional files 1, 2, 3 and 4.

\section{About this supplement}

This article has been published as part of BMC Medical Genomics Volume 12 Supplement 1, 2019: Selected articles from the International Conference on Intelligent Biology and Medicine (ICIBM) 2018: medical genomics. The full contents of the supplement are available online at https:// bmcmedgenomics.biomedcentral.com/articles/supplements/volume-12supplement-1. 


\section{Authors' contributions}

CNVs were mapped and annotated to genes by EL. PC collected the patient data for sarcoma disease prognostic associated genes. Public data was collected and analyzed by LC. CNVs associated with drug response in cell lines was analyzed by LW. LL, LC, PHP, KEP, MEM, MM, JC, MF, MRS, KB, and $J L R$ contributed to the writing and editing of the manuscript. All authors read and approved the final manuscript.

\section{Ethics approval and consent to participate \\ Not applicable}

\section{Consent for publication}

Not applicable

\section{Competing interests}

The authors declare that they have no competing interests.

\section{Publisher's Note}

Springer Nature remains neutral with regard to jurisdictional claims in published maps and institutional affiliations.

\section{Author details}

${ }^{1}$ Department of Biomedical Informatics, College of Medicine, Ohio State University, Columbus, OH 43210, USA. ${ }^{2}$ Herman B Wells Center for Pediatric Research, Department of Pediatrics, School of Medicine, Indiana University, Indianapolis, IN 46202, USA. Division of Hematology/Oncology, Department of Pediatrics, School of Medicine, Indiana University, Indianapolis, IN 46202, USA. ${ }^{4}$ Center for Computational Biology and Bioinformatics, School of Medicine, Indiana University, Indianapolis, IN 46202, USA. ${ }^{5}$ Indiana University Melvin and Bren Simon Cancer Center, Indianapolis, IN 46202, USA. ${ }^{6}$ Indiana Institute of Personalized Medicine, Indiana University, Indianapolis, IN 46202, USA

\section{Published: 31 January 2019}

\section{References}

1. HaDuong JH, Martin AA, Skapek SX, Mascarenhas L. Sarcomas. Pediatr Clin N Am. 2015;62(1):179-200.

2. Tirode F, Surdez D, Ma X, Parker M, Le Deley MC, Bahrami A, Zhang Z Lapouble E, Grossetete-Lalami S, Rusch M, et al. Genomic landscape of Ewing sarcoma defines an aggressive subtype with co-association of STAG2 and TP53 mutations. Cancer discov. 2014;4(11):1342-53.

3. Shern JF, Chen L, Chmielecki J, Wei JS, Patidar R, Rosenberg M, Ambrogio L, Auclair D, Wang J, Song YK, et al. Comprehensive genomic analysis of rhabdomyosarcoma reveals a landscape of alterations affecting a common genetic axis in fusion-positive and fusion-negative tumors. Cancer discov. 2014;4(2):216-31

4. Crompton BD, Stewart C, Taylor-Weiner A, Alexe G, Kurek KC, Calicchio ML, Kiezun A, Carter SL, Shukla SA, Mehta SS, et al. The genomic landscape of pediatric Ewing sarcoma. Cancer discov. 2014:4(11):1316-41.

5. Martin JW, Squire JA, Zielenska M. The genetics of osteosarcoma. Sarcoma. 2012;2012:11.

6. Potratz J, Jurgens H, Craft A, Dirksen U. Ewing sarcoma: biology-based therapeutic perspectives. Pediatr Hematol Oncol. 2012;29(1):12-27.

7. Morrow JJ, Khanna C. Osteosarcoma genetics and epigenetics: emerging biology and candidate therapies. Crit Rev Oncog. 2015;20(3-4):173-97.

8. Taylor BS, Barretina J, Maki RG, Antonescu CR, Singer S, Ladanyi M. Advances in sarcoma genomics and new therapeutic targets. Nat Rev Cancer. 2011; 11(8):541-57.

9. Theisen ER, Pishas KI, Saund RS, Lessnick SL. Therapeutic opportunities in Ewing sarcoma: EWS-FLI inhibition via LSD1 targeting. Oncotarget. 2016; 7(14):17616-30.

10. Smida J, Xu H, Zhang Y, Baumhoer D, Ribi S, Kovac M, von Luettichau I, Bielack S, O'Leary VB, Leib-Mosch C, et al. Genome-wide analysis of somatic copy number alterations and chromosomal breakages in osteosarcoma. Int J Cancer. 2017:141(4):816-28.

11. van Dartel M, Hulsebos TJ. Amplification and overexpression of genes in 17p11.2 p12 in osteosarcoma. Cancer Genet Cytogenet. 2004;153(1):77-80.

12. Sun X, Guo W, Shen JK, Mankin HJ, Hornicek FJ, Duan Z. Rhabdomyosarcoma: advances in molecular and cellular biology. Sarcoma. 2015:2015:232010
13. Chaiyawat P, Settakorn J, Sangsin A, Teeyakasem P, Klangjorhor J, Soongkhaw A, Pruksakorn D. Exploring targeted therapy of osteosarcoma using proteomics data. Onco Targets Ther. 2017;10:565-77.

14. Chen X, Bahrami A, Pappo A, Easton J, Dalton J, Hedlund E, Ellison D, Shurtleff S, Wu G, Wei L, et al. Recurrent somatic structural variations contribute to tumorigenesis in pediatric osteosarcoma. Cell Rep. 2014;7(1): 104-12.

15. Specht K, Sung YS, Zhang L, Richter GH, Fletcher CD, Antonescu CR. Distinct transcriptional signature and immunoprofile of CIC-DUX4 fusion-positive round cell tumors compared to EWSR1-rearranged Ewing sarcomas: further evidence toward distinct pathologic entities. Genes, chromosomes cancer. 2014;53(7):622-33

16. Lynn M, Wang Y, Slater J, Shah N, Conroy J, Ennis S, Morris T, Betts DR, Fletcher JA, O'Sullivan MJ. High-resolution genome-wide copy-number analyses identify localized copy-number alterations in Ewing sarcoma. Diagn Mol Pathol. 2013;22(2):76-84.

17. Lynn M, Shah N, Conroy J, Ennis S, Morris T, Betts D, O'Sullivan M. A study of alveolar rhabdomyosarcoma copy number alterations by single nucleotide polymorphism analysis. Appl Immunohistochem Mol Morphol. 2014;22(3):213-21.

18. Liu C, Li D, Jiang J, Hu J, Zhang W, Chen Y, Cui X, Qi Y, Zou H, Zhang W, et al. Analysis of molecular cytogenetic alteration in rhabdomyosarcoma by array comparative genomic hybridization. PLoS One. 2014;9(4):e94924.

19. Kallioniemi A. CGH microarrays and cancer. Curr Opin Biotechnol. 2008;19(1):36-40

20. MacDonald JR, Ziman R, Yuen RK, Feuk L, Scherer SW. The database of genomic variants: a curated collection of structural variation in the human genome. Nucleic Acids Res. 2014;42(Database issue):D986-92.

21. Garnett MJ, Edelman EJ, Heidorn SJ, Greenman CD, Dastur A, Lau KW, Greninger P, Thompson IR, Luo X, Soares J, et al. Systematic identification of genomic markers of drug sensitivity in cancer cells. Nature. 2012;483(7391): $570-5$.

22. Seashore-Ludlow B, Rees MG, Cheah JH, Cokol M, Price EV, Coletti ME, Jones V, Bodycombe NE, Soule CK, Gould J, et al. Harnessing connectivity in a large-scale small-molecule sensitivity dataset. Cancer discov. 2015;5(11): $1210-23$.

23. Kuijjer ML, Rydbeck $H$, Kresse $S H$, Buddingh EP, Lid AB, Roelofs $H$, Burger $H$, Myklebost O, Hogendoorn PC, Meza-Zepeda LA, et al. Identification of osteosarcoma driver genes by integrative analysis of copy number and gene expression data. Genes, chromosomes cancer. 2012;51(7):696-706.

24. Kovac M, Blattmann C, Ribi S, Smida J, Mueller NS, Engert F, Castro-Giner F, Weischenfeldt J, Kovacova M, Krieg A, et al. Exome sequencing of osteosarcoma reveals mutation signatures reminiscent of BRCA deficiency. Nat Commun. 2015;6:8940.

25. Moriarity BS, Otto GM, Rahrmann EP, Rathe SK, Wolf NK, Weg MT, Manlove LA, LaRue RS, Temiz NA, Molyneux SD, et al. A sleeping beauty forward genetic screen identifies new genes and pathways driving osteosarcoma development and metastasis. Nat Genet. 2015;47(6):615-24

26. Behjati S, Tarpey PS, Haase K, Ye H, Young MD, Alexandrov LB, Farndon S, Collord G, Wedge DC, Martincorena I, et al. Recurrent mutation of IGF signalling genes and distinct patterns of genomic rearrangement in osteosarcoma. Nat Commun. 2017;8:15936.

27. Agelopoulos K, Richter GH, Schmidt E, Dirksen U, von Heyking K, Moser B, Klein HU, Kontny $U$, Dugas M, Poos K, et al. Deep sequencing in conjunction with expression and functional analyses reveals activation of FGFR1 in Ewing sarcoma. Clin Cancer Res. 2015;21(21):4935-46.

28. Ferreira BI, Alonso J, Carrillo J, Acquadro F, Largo C, Suela J, Teixeira MR, Cerveira N, Molares A, Gomez-Lopez G, et al. Array CGH and geneexpression profiling reveals distinct genomic instability patterns associated with DNA repair and cell-cycle checkpoint pathways in Ewing's sarcoma. Oncogene. 2008;27(14):2084-90.

29. Jahromi MS, Jones KB, Schiffman JD. Copy number alterations and methylation in Ewing's sarcoma. Sarcoma. 2011;2011:10.

30. Nishimura R, Takita J, Sato-Otsubo A, Kato M, Koh K, Hanada R, Tanaka Y, Kato K, Maeda D, Fukayama M, et al. Characterization of genetic lesions in rhabdomyosarcoma using a high-density single nucleotide polymorphism array. Cancer Sci. 2013;104(7):856-64.

31. Seki M, Nishimura $R$, Yoshida $K$, Shimamura $T$, Shiraishi $Y$, Sato $Y$, Kato M, Chiba K, Tanaka H, Hoshino N, et al. Integrated genetic and epigenetic analysis defines novel molecular subgroups in rhabdomyosarcoma. Nat Commun. 2015:6:7557. 
32. Zhang Z, Zhang L, Hua Y, Jia X, Li J, Hu S, Peng X, Yang P, Sun M, Ma F, et al. Comparative proteomic analysis of plasma membrane proteins between human osteosarcoma and normal osteoblastic cell lines. BMC Cancer. 2010:10:206.

33. Walia MK, Ho PM, Taylor S, Ng AJ, Gupte A, Chalk AM, Zannettino AC, Martin TJ, Walkley CR. Activation of PTHrP-CAMP-CREB1 signaling following p53 loss is essential for osteosarcoma initiation and maintenance. elife. 2016;5.

34. Kansara M, Teng MW, Smyth MJ, Thomas DM. Translational biology of osteosarcoma. Nat Rev Cancer. 2014;14(11):722-35.

35. Perry JA, Kiezun A, Tonzi P, Van Allen EM, Carter SL, Baca SC, Cowley GS, Bhatt AS, Rheinbay E, Pedamallu CS, et al. Complementary genomic approaches highlight the PI3K/mTOR pathway as a common vulnerability in osteosarcoma. Proc Natl Acad Sci U S A. 2014;111(51):E5564-73.

36. Kalkat M, De Melo J, Hickman KA, Lourenco C, Redel C, Resetca D, Tamachi A, Tu WB, Penn LZ. MYC deregulation in primary human cancers. Genes. 2017:8(6).

37. Xiong $Y$, Wu S, Du Q, Wang A, Wang Z. Integrated analysis of gene expression and genomic aberration data in osteosarcoma (OS). Cancer Gene Ther. 2015;22(11):524-9.

38. Quinlan AR, Ira MH. BEDTools: a flexible suite of utilities for comparing genomic features. Bioinformatics. 2010;16(6):841-2.

39. Steven AM, Finny GK, David A. Integrated detection and population-genetic analysis of SNPs and copy number variation. Nat Genet. 2008;40:1166-74.

40. Andrew SB, David AS, Wendy C, Jianjun W, Young S, Sivasish S, Rajesh P, Laura H, Li C, Jack FS, Hongling L, Xinyu W, Julia G, Javed K. The genomic landscape of the Ewing sarcoma family of tumors reveals recurrent STAG2 mutation. PLoS Genet. 2014;10(8):e1004629.

41. Su Young K, Jeffrey AT, Daniel S, Lee JH. The role of IGF-1R in pediatric malignancies. Oncologist. 2009;14(1):83-91.

42. Todd MC, Bassem IR, Robert Gerbing MA, Todd AA, Kathleen Adlard RN, Elizabeth R, Alan SG, John P, James AW. Phase I/II trial of Clofarabine and Cytarabine in children WithRelapsed/refractory acute lymphoblastic leukemia (AAML0523):a report from the Children's oncology group. Pediatr Blood Cancer. 2013;60:1141-7.

43. Vasudevaraja V, Renbarger J, Shah RG, Kinnebrew G, Korc M, Wang L, Cheng L. PMTDS: a computational method based on genetic interaction networks for precision medicine target-drug selection in cancer. Quantitative Biology. 2017;5(4):380-94

44. Subbiah V, Wagner MJ, McGuire MF, Sarwari NM, Devarajan E, Lewis VO, Westin S, Kato S, Brown RE, Anderson P. Personalized comprehensive molecular profiling of high risk osteosarcoma: implications and limitations for precision medicine. Oncotarget. 2015;6(38):40642-54.

45. Zerbino DR, Achuthan P, Akanni W, Amode MR, Barrell D, Bhai J, Billis $K$, Cummins C, Gall A, Girón CG, et al. Ensembl 2018. Nucleic Acids Res. 2018;46(D1):D754-61.

46. Bruford EA, Lush MJ, Wright MW, Sneddon TP, Povey S, Birney E. The HGNC database in 2008: a resource for the human genome. Nucleic Acids Res. 2008:36(Database issue):D445-8.

Ready to submit your research? Choose BMC and benefit from:

- fast, convenient online submission

- thorough peer review by experienced researchers in your field

- rapid publication on acceptance

- support for research data, including large and complex data types

- gold Open Access which fosters wider collaboration and increased citations

- maximum visibility for your research: over $100 \mathrm{M}$ website views per year

At $\mathrm{BMC}$, research is always in progress.

Learn more biomedcentral.com/submissions 